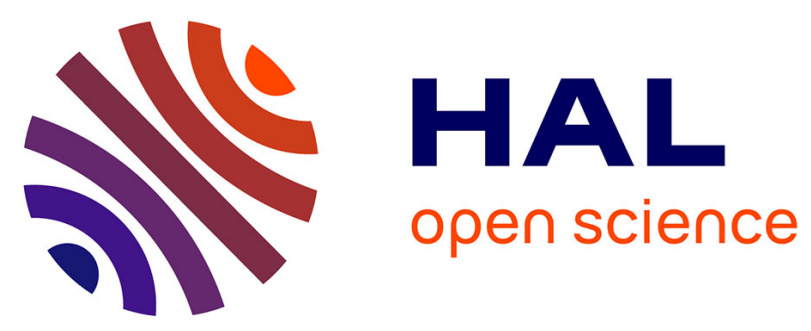

\title{
Review of Terahertz Tomography Techniques
}

\author{
Jean Paul Guillet, Benoît Recur, Louis Frederique, Bruno Bousquet, Lionel
}

Canioni, Inka Manek-Hönninger, Pascal Desbarats, Patrick Mounaix

\section{To cite this version:}

Jean Paul Guillet, Benoît Recur, Louis Frederique, Bruno Bousquet, Lionel Canioni, et al.. Review of Terahertz Tomography Techniques. Journal of Infrared, Millimeter and Terahertz Waves, 2014, 35 (4), pp.382-411. 10.1007/s10762-014-0057-0 . hal-00968839

\section{HAL Id: hal-00968839 \\ https://hal.science/hal-00968839}

Submitted on 1 Apr 2014

HAL is a multi-disciplinary open access archive for the deposit and dissemination of scientific research documents, whether they are published or not. The documents may come from teaching and research institutions in France or abroad, or from public or private research centers.
L'archive ouverte pluridisciplinaire HAL, est destinée au dépôt et à la diffusion de documents scientifiques de niveau recherche, publiés ou non, émanant des établissements d'enseignement et de recherche français ou étrangers, des laboratoires publics ou privés.

\section{다)(1) $(5$}

Distributed under a Creative Commons Attribution - NonCommerciall 4.0 International 


\title{
Review of Terahertz Tomography Techniques
}

\author{
J. P. Guillet • B. Recur • L. Frederique • B. Bousquet • L. Canioni • \\ I Manek-Hönninger $\cdot$ P. Desbarats $・ P$. Mounaix
}

\begin{abstract}
Terahertz and millimeter waves penetrate various dielectric materials, including plastics, ceramics, crystals, and concrete, allowing terahertz transmission and reflection images to be considered as a new imaging tool complementary to X-Ray or Infrared. Terahertz imaging is a well-established technique in various laboratory and industrial applications. However, these images are often two-dimensional. Three-dimensional, transmission-mode imaging is limited to thin samples, due to the absorption of the sample accumulated in the propagation direction. A tomographic imaging procedure can be used to acquire and to render three-dimensional images in the terahertz frequency range, as in the optical, infrared or X-ray regions of the electromagnetic spectrum. In this paper, after a brief introduction to two dimensional millimeter waves and terahertz imaging we establish the principles of tomography for Terahertz Computed tomography (CT), tomosynthesis (TS), synthetic aperture radar (SAR) and time-of-flight (TOF) terahertz tomography. For each technique, we present advantages, drawbacks and limitations for imaging the internal structure of an object.
\end{abstract}

Keywords Tomography - Terahertz imaging $\cdot 3 \mathrm{D}$ reconstruction - Time of flight $\cdot$ Holography · Diffractive imaging $\cdot$ Synthetic aperture radar $(\mathrm{SAR}) \cdot \mathrm{THz}$ Computed tomography $\cdot$ Iterative method · BFP · Volume inspection · Non destructive testing · Sinogram · Millimeter waves · Far infrared $\cdot$ Spectro imaging $\cdot$ Reflection and transmission images

\section{Introduction to 2D Imaging Techniques and Application Fields}

Terahertz radiation $\left(1 \mathrm{THz}=10^{12} \mathrm{~Hz}\right)$ are electromagnetic waves in the spectral range going from 0.3 to $10 \mathrm{THz}$. Low energy interaction of a wide range of materials can thus be studied by these waves and new data can potentially be retrieved to complete the knowledge of material behavior with respect to far infrared (FIR) and Raman spectroscopy. Terahertz technology has

J. P. Guillet $\cdot$ B. Recur $・$ B. Bousquet $\cdot$ L. Canioni $\cdot$ I. Manek-Hönninger $\cdot$ P. Mounaix $(\bowtie)$

Université de Bordeaux, LOMA, UMR CNRS 5798, 351 Cours de la Libération, 33405 Talence Cedex, France

e-mail: p.mounaix@loma.u-bordeaux1.fr

L. Frederique $\cdot$ P. Desbarats

Université de Bordeaux, LaBRI, UMR CNRS 5800, 351 Cours de la Libération, 33405 Talence Cedex, France 
been making clear progress following recent advances in femtosecond lasers and ultrafast semiconductor coherent sources emitting in the $\mathrm{THz}$ frequency range [1]. However, the development of brighter sources and efficient detectors that are more sensitive to $\mathrm{THz}$ waves is a key feature to efficiently use these waves in THz spectroscopy [2]. One of the most interesting properties of $\mathrm{THz}$ waves is their capability to transmit through an extensive variety of materials. Thus they can be used to test, image [3] or analyze samples through many types of covering materials, such as paper and plastics, and allow imaging in a standoff configuration $[4,5]$. Moreover, the interaction of a substance with $\mathrm{THz}$ waves is non destructive, and thus these radiations can also be used to probe fragile biological materials [6-9], for medical diagnostics or bio medical imaging [10-13]. These applications are not limited to basic research and can be applied in a wide variety of fields.

Ranging between far infrared and microwaves, the development of terahertz science and technology [14, 15] comes from both the electronics [16, 17] and optics sides [18]. Recent innovations in terahertz technology take advantages from two outstanding ideas and concepts from each individual field. More recently, the inventory of applications has increased and includes quality control, non destructive testing (NDT) [19-21] and spectroscopic characterization of materials $[22,23]$ or chemical recognition [24]. Many of these applications rely on the exceptional features of terahertz radiation which consist of the sufficient transparency of materials and the fact that many interesting chemical compounds display unique spectral fingerprints in the terahertz range. This advantage can be exploited in industrial applications [25], for identification or recognition and chemical analysis [26, 27].

Since two-dimensional $\mathrm{THz}$ transmission images were first acquired by $\mathrm{Hu}$ and Nuss in 1995 [28], the revolutionary work of Zhang and co-workers [29, 30], who developed fast electro optic detection at video rate for imaging purpose [31], sounded the beginning of 2D and 3D imaging science. Afterward, developments in terahertz imaging could use either a terahertz time-domain spectroscopy modified setup (terahertz radiation is generated in the form of short single-cycle pulses and large spectral bandwidth) or a narrow band terahertz generator coming from the microwave world, semiconductor technology or optics development. This progress has occurred simultaneously with numerous efficient sources and detectors for continuous-wave $(\mathrm{cw})$ terahertz radiation [32-34]. Now scientists are able to propose optimized solutions of terahertz imaging for a given application with an optimal setup configuration [35] (e.g. pulsed or continuous generator, broadband versus narrowband, spectral resolution versus temporal resolution, active versus passive imaging [36], but also near field [37-40], wave guiding [41], detectors array and even more camera [42], etc.).

Before the description of 3D imaging techniques, we will briefly present $2 \mathrm{D}$ imaging results and capabilities that rely both on time-domain systems and other terahertz sources. Significant technological advancements have been reviewed in several scientific papers [43-48]. It is worth considering the present description from results on millimeter-wave imaging and describing on imaging applications with terahertz radiation. Some typical references are provided for the interested reader.

\section{Time Domain Techniques}

The performance characteristics of THz Time Domain Systems (THz-TDS) have been reviewed in many previous publications [47-55] and books [56-61], and will not be discussed in this paper. However, we will give a very succinct description of the spectroscopic technique, and some particular features or limitations for imaging development. 
THz-TDS is the most commonly used technique. In a typical setup, THz-TDS enables the determination of the complex permittivity of a sample over a frequency range of typically $0.1-4 \mathrm{THz}$. The analysis can be conducted in transmission or reflection modes and provides information about the static properties of the sample over several frequency decades [62]. Briefly, there are two key mechanisms for the generation and detection of $\mathrm{THz}$ pulses. Both take advantages of a nonlinear process when optically exciting a suitable material: photoninduced conductivity (photoconductivity) in an ultrafast semiconductor. In this case, electronhole pairs are accelerated by a static field applied into the active device, the consequent transient current into the antenna due to charge separation induces the electromagnetic generation in the environment space. The second type of interaction, non-resonant, is the well known optical rectification in crystals presenting high electro-optic coefficients. Although the physical mechanism of $\mathrm{THz}$ generation using nonlinear optics differs considerably from the one used in photoconductive generation, both finally drive to a generation of a transient polarization. Since the generated polarization does not rely on carrier transport, extremely high bandwidth pulses can be generated, limited only by the duration and bandwidth of the exciting optical pulse and phase matching conditions.

Due to the very short time scale of the generated electric field, optical sampling techniques are employed assuming that all the $\mathrm{THz}$ pulses are identical. A second possibility for $\mathrm{THz}$ detection is based on the electro-optic effect in a crystal, which is due to the coupling between a low frequency electric field (THz pulse) and a laser beam (optical pulse) [63]. Free-space electro-optic sampling via the linear electro-optic effect (Pockels effect) offers a flat frequency response over an ultra wide spectral bandwidth, mainly limited by either the pulse duration of the probe laser or the lowest Transverse optical (TO) phonon frequency of the sensor crystal. Since electro-optic sampling is solely an optical procedure, it does not require any technological facilities for mounting the active devices [64].

Based on this coherent detection of the THz field [65], the average photo induced current is a replicate of the $\mathrm{THz}$ field variations as a function of time, and consequently, the temporal -or spectral- transmitted or reflected $\mathrm{THz}$ beams could be used to construct a contrast image stepby-step [66] as illustrated in Fig.1

Despite numerous advantages, imaging with a THz-TDS system presents some limitations [32]. First of all, the generated power in the THz beam is quite small (less than a dozen $\mu \mathrm{W}$ average power), so it must be associated to very high dynamic range [67] sensors and techniques. This high dynamic range allows successful measurement even with a small amount of power. However, since no efficient 2D coherent detectors are available, most of the time-domain imaging systems are based on scanning of the sample and reconstruction of a
A: Maximum sample,
B: Minimum sample,
C: Amplitude sample,
D: Absorption line
E: Max FFT Amplitude
a: Max Peak delay,
b: Delay min sample,
c: Zero peak delay,
e: Max of FFT,
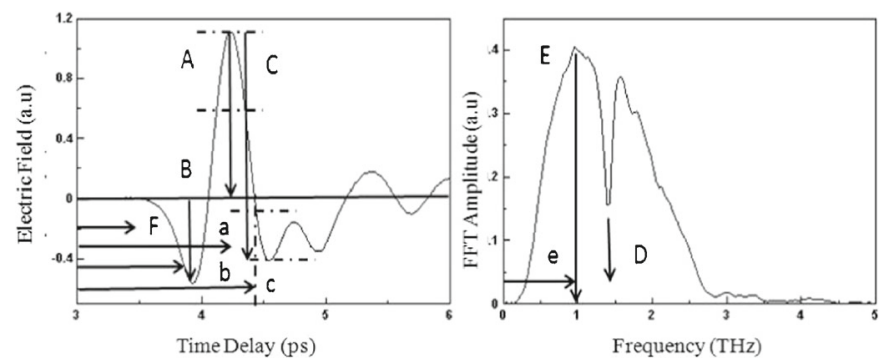

Figure 1 displays an example of plots in time and frequency domains including specific parameters which can be chosen for contrast features to build a $2 \mathrm{D}$ terahertz image pixel by pixel. 
series of single-point measurements. The main drawback is then a rather slow image acquisition rate which is not suitable for many applications.

Another problem is the sampling technique used to reconstruct the time response of the reflected or transmitted beam. The spectral resolution is linked to the inverse of the temporal window during the acquisition step. So there is a clear limitation with time necessitating a tradeoff between duration and spectral resolution.

For image acquisition, the highest scan rates (e.g. from hundreds to thousands of temporal data points per second) were achieved using a piezo-electric device, but with a limited (tens of picosecond) temporal range and consequently a limited spectral resolution. At such high rates, the total duration for image acquisition is no longer limited by the measurement of the $\mathrm{THz}$ waveform, but by the motion speed of the sample. In addition, a shorter scanning range also limits the penetration depth of the THz pulse into a sample. Different categories of motorized scanning devices (e.g. spinning or vibrating mirror devices) can produce several hundreds of picoseconds of delay range with a high scanning rate typically more than few hundreds of hertz. Recently, very promising results were published about the design of compact and inexpensive terahertz imaging systems relying on femtosecond fiber laser technology without mechanical stage (Ecops [68], Asops [69], OSCAT [70]). In these cases, the motion of the scanning delay line must be synchronized to the raster scan of the object, so that it is possible to determine the location of the object at the moment each waveform is acquired. Recently a single pixel imaging system has been proposed based on a compressed sensing approach [71] This system does not rely on raster scanning or a source/detector array, but uses random patterns for imaging.

Since the first images with a THz-TDS system were obtained, a huge number of possibilities have been published in the literature with electronic sources. For example, the source could be a quantum cascade laser [72], Gunn diode [73], plasma wave transistor [74, 75], Backward Wave Oscillator [76], and this list in non exhaustive. We also notice the use of high power sources [77], CW gas laser [78], coherent or incoherent detectors and any combination of all these devices [79]. Moreover, a lot of new techniques have emerged such as polarization imaging [80] dark-field imaging [81], single-pixel imaging [82-84] and compressive imaging technique[85], real time imaging [86], time reversal imaging [87], terahertz microscope [88, 89] and interferometric imaging $[90,91]$. This is just to mention that terahertz imaging has given rise to a lot of attention and developments during this last decade.

\section{Examples of Application Fields}

\subsection{Security Application}

Among security applications of THz imaging [92], let's point out the inspection of luggage (example fig.2) and postal mail [93]. The main idea relies on the fact that molecular crystals present very specific features in the THz spectrum. For example, explosives [94-97] or illicit drugs [98-100] have been efficiently localized and unambiguously identified within an envelope [101], a parcel, or a suitcase [102]. Besides, dangerous metallic items like guns [103] or knifes become clearly visible by their shapes and can be easily identified by pattern recognition algorithm. Yet, obviously metallic packages are opaque to $\mathrm{THz}$ waves. For this reason, THz spectrometers are not likely to substitute X-ray scanners. However, they can provide supplementary information on the sample, mainly for low density materials and chemical separation. Other interesting applications concern liquid explosives. Several liquids show very different dielectric response in the terahertz range versus their alcohol percentage for example and can thus be distinguished [104]. 

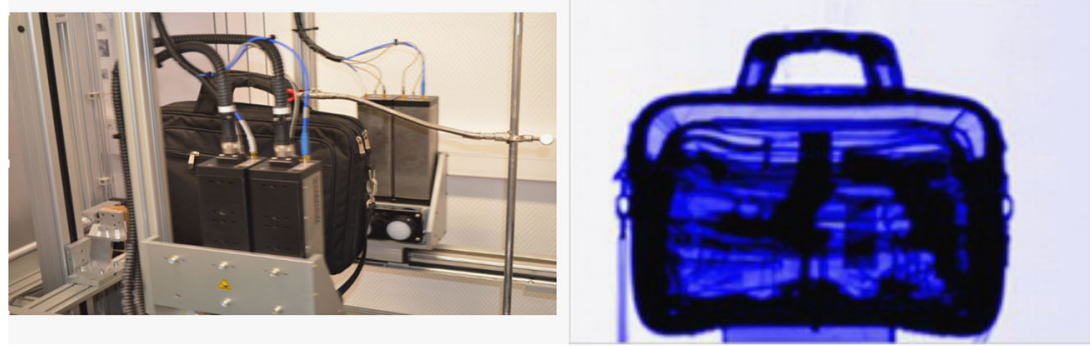

Figure 2 100GHz transmission image through a computer bag : two guns are clearly visible (LOMA-Univ. Bordeaux unpublished)

\subsection{Paper and Polymer Industries}

On-line production control can be efficiently done by $\mathrm{THz}$ systems in the paper and polymer industries [105]. In the case of paper, both the thickness and moisture content are monitored during the production process. Mousavi and coworkers [106] demonstrated that two different paper samples could be differentiated by $\mathrm{THz}$ with similar accuracy to other sensors. In the polymer industry online supervision of polymeric processes (for example a real time paint meter [107]), quality control of plastic weld joints [108], conductive properties [109], determination of moisture level [110], the fiber orientation [111] and the glass-transition temperature of polymers have been demonstrated by $\mathrm{THz}$ systems [112].

\subsection{Food Industry}

The detection of unwanted and potentially hazardous objects in food is extremely important in the food industry. Both metallic and non metallic contaminations can be detected by $\mathrm{THz}$ systems [113]. Additionally low water content foodstuff such as chocolate is transparent enough to $\mathrm{THz}$ waves to enable Jördens and Koch [114] to detect the presence of different metallic and nonmetallic inclusions within chocolate bars. The study of moisture quantification within an industrial process could be interesting; for instance in the fresh food industry with packaging protection. For wine or alcohol making company, cork substance is important to qualify. Their structure, and low moisture content are reasonably transparent to terahertz $(\mathrm{THz})$. Nondestructive evaluation of the cork's surface and interior has been done [115] and the contrast in the THz images is a result of enhanced scattering of $\mathrm{THz}$ radiation by defects or voids as well as variations in the cork cell structure as shown in figure 3 extracted from [115].

\subsection{Pharmaceutical Industry}

The potential of THz systems in the pharmaceutical industry was recently highlighted [116, 117]. Since different isomers lead to crystalline structures with varying spectra fingerprints in the $\mathrm{THz}$ range, polymorphic forms can be detected. This detection is much appreciated since the chirality [113] is a major concern when dealing with the pharmaceutical performance of the active principle [118]. Moreover, THz is well suited for quality control of tablet coatings [119] that are used to control the release of active pharmaceutical ingredients (see Fig.4) [120-122]. A review of this application field has been published [117]. 

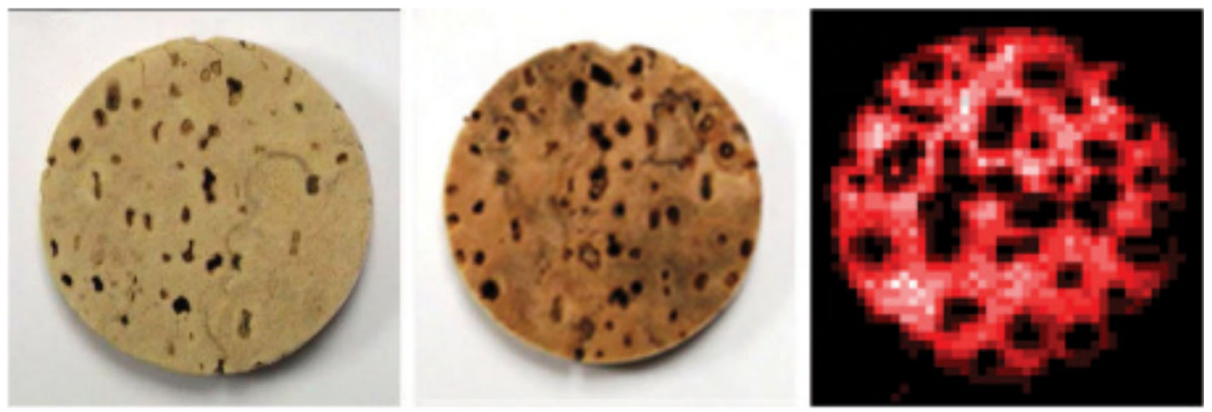

Figure 3 Evaluation of cork enclosures by terahertz imaging taken from [115]

\subsection{Art Conservation}

The importance of the knowledge of materials and underlying materials science is crucial in optimizing preservation of cultural heritage artwork. For terahertz science the challenges faced in the development of non-destructive analytical methods and its integration with new diagnostic techniques are numerous. For these new tools, it's important to be portable on site and to be safe for the sample under investigation. However, heritage materials diagnostics [123] is difficult: not only because the sampling of artworks is usually not allowed, but also because the measurement is generally required to be done on-site. To illustrate this purpose, Jackson et al. performed THz investigation of mural paintings [124]. Using THz-TDS, they revealed the presence of a graphite drawing [125] under layers of paint and plaster. Concerning painting, Adam and coworkers compared $\mathrm{THz}$ reflection images of hidden paint layers [126]. A similar study was presented by Fukunaga and coworkers [127] to quantify the transmittance of several pigments. Recently, they extended their work to a medieval manuscript [128] and even mummies have been studied by $\mathrm{THz}$ imaging [129]. All this clearly demonstrates the potential of THz imaging for Art and Archeological science (Fig.5).

Terahertz (THz) spectroscopy associated with $\mathrm{THz}$ imaging, holds large potential in the field of non destructive, contact-free testing. The constant advances in the development of $\mathrm{THz}$ systems, as well as the appearance of the first related commercial products, indicate that large-

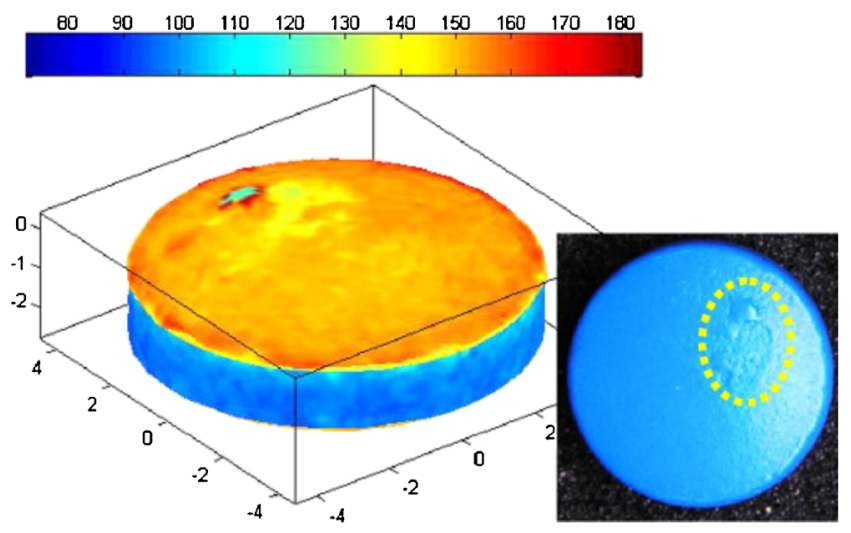

Figure 4 3D images of the two tablet surfaces and the central band. The color coded bar represents coating layer thickness, the scale is in microns [122]. The coating layer thickness around the central band is much thinner than that on the surfaces of the tablet 
a Visible image

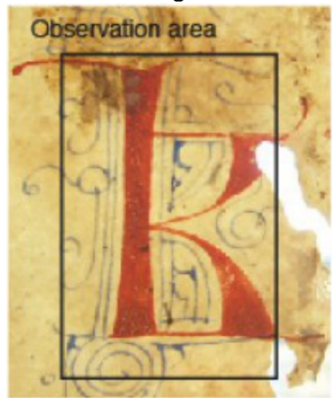

c Extracted mapping of red ink

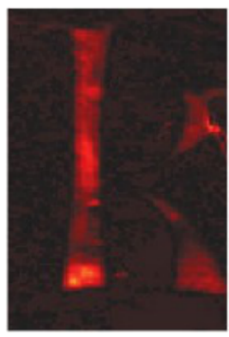

b Extracted mapping of

base parchment

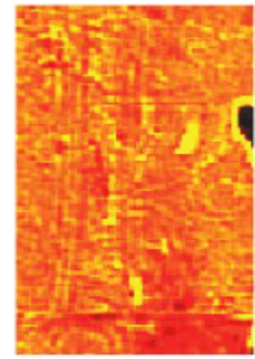

d Extracted mapping

of stain

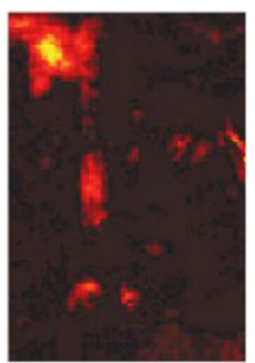

Figure 5 Images of a part of a medieval parchment with visible and terahertz light [128]

scale market introduction of $\mathrm{THz}$ systems is rapidly approaching. Nowadays, there is a considerable interest in tomographic methods for THz imaging $[130,131]$ by adding the third dimension for analyzing different samples. Short pulses of broadband THz radiation [132], continuous wave sources and microwaves [133] are used to illuminate the target [134]. Coherent detection methods are used to allow the reflected or transmitted $\mathrm{THz}$ pulse profile to be measured [135].

\section{Tomographic Imaging}

The word tomography is derived from the Greek word tomos meaning 'slice' or 'section' and graphia meaning 'describing'. The field of tomography involves methods for obtaining cross sectional images of a target, allowing the internal detail to be observed.

In recent years, a number of methods for $3 \mathrm{D}$ imaging with $\mathrm{THz}$ radiation have been proposed and demonstrated [136]. Since the development of pulsed THz systems, such 3D imaging systems have been proposed. Let's describe briefly the different physical techniques capable of extracting 3D information.

\subsection{THz Diffraction Tomography}

The goal of diffraction tomography is to determine the spatial distribution of a sample's refractive index using the measurement of the diffracted $\mathrm{THz}$ field. The relationship between the $\mathrm{THz}$ wave distribution and the object's refractive index as a function of position can be described by the Maxwell's equations. The basis of diffraction tomography is to linearize inverse scattering equations based on several hypotheses commonly used in ultrasound tomography systems. In diffraction tomography, a probe beam interacts with a target and then the 3D image of the sample is built using the waves scattered by the target [137]. This is the main difference with Computed Tomography (CT) which generally utilizes the amplitude signal directly transmitted through the sample. A THz diffraction tomography signal contains the same information as the CT signal and finally the target structural information in $3 \mathrm{D}$ is measured and processed by the diffracted $\mathrm{THz}$ distribution. This technique is more adapted to complex samples with fine structures in which diffraction effects dominate the measurements. The first implementation of this method was reported by Ferguson et al. who imaged a simple polyethylene cylinder and proposed a reconstruction method based on the Born linearization of the wave equation [138]. 
Wang et al. performed $\mathrm{THz}$ diffraction tomography by using a mode-locked femtosecond laser, generating $\mathrm{THz}$ radiation by optical rectification in a ZnTe crystal and detecting the signal with a CCD camera [132] as illustrated in fig.6. They proposed a reconstruction method based on the electromagnetic wave scattering theory. Three rectangular polyethylene cylinders $(n=1.5)$ were imaged by using the $\mathrm{THz}$ diffraction pattern at various projection angles. The application of $\mathrm{THz}$ diffraction tomography was able to provide the refractive index distribution of the target. A comparison of image quality as a function of the $\mathrm{THz}$ frequency was also carried out. $\mathrm{THz}$ diffraction tomography often provides poor reconstructed images owing to the problem of suitable reconstruction algorithms and signal interpretation [139, 140]. The reconstruction is hindered by the relatively low signal to noise ratio of the $\mathrm{THz}$ field measurements. Moreover, these methods assume that the target is dispersionless, which removes a key advantage of $\mathrm{THz}$ techniques, namely the spectroscopic information extraction. However, the image acquisition speed of $\mathrm{THz}$ diffraction tomography is relatively faster than $\mathrm{THz} \mathrm{CT}$. $\mathrm{THz}$ diffraction tomography reconstructs the inverse of target's refractive index distribution.

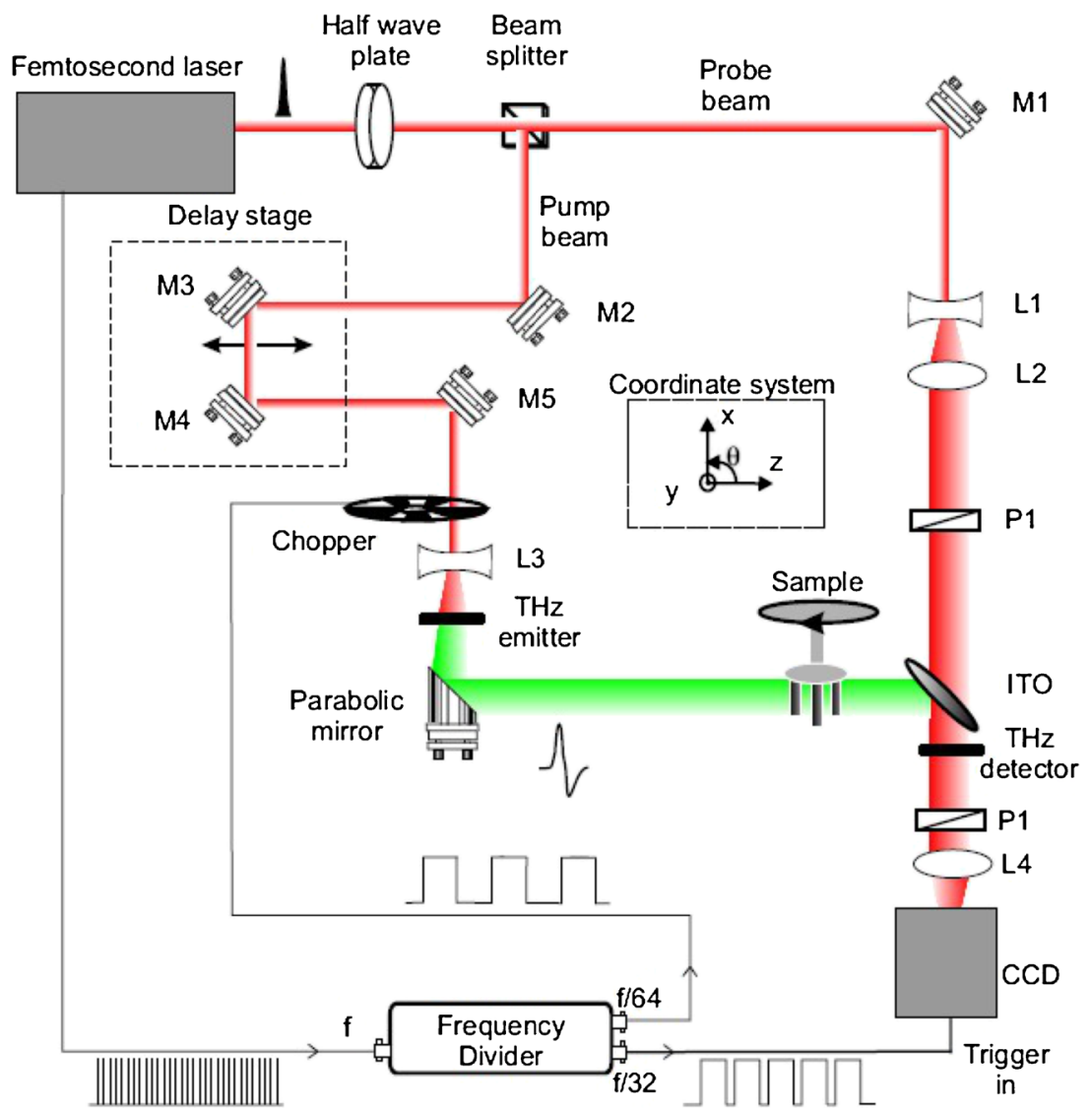

Figure 6 Setup up for diffraction tomography taken from [132]. 


\subsection{THz Tomosynthesis}

Tomosynthesis (TS) is a straightforward but useful method for reconstructing few slices of a 3D object from a limited number of projections [141]. It does not require measurements in all directions, and can reconstruct tomographic images using only projections within small angles. It is commonly used for medical imaging for breast cancer detection for example [142]. However, unlike tomography where projections are measured all around the object, in tomosynthesis, they are done according to a limited angle range (usually between $-50^{\circ}$ and $50^{\circ}$ ). Figure 7 shows the principle of this acquisition system.

Projections (or radiographs) are made at different points of view with a moving source along a linear trajectory. A detector plane is opposed to the emitter plane and measures the attenuation of the $\mathrm{THz}$ transmission. A series of radiographs of the object are obtained according to different points of view.

This technique could retrieve a layer of interest against the out-of-focus layers. This method typically employs absorption images of an object and the different projections from various views, similar to a standard computed tomography. However, as mentioned, tomosynthesis relies on a much lower number of views at selected angles, which can be seen as computed tomography with missing information. Fig.7 shows that performing a large shifting of the superposed radiographs enforces the signal corresponding to the little circle in grey (for the left image). On the contrary, a small shifting allows focusing on the $\mathrm{x}-\mathrm{y}$ plane at the $\mathrm{z}$ depth where the dark grey object is positioned (for the right image). Because of the similarity between tomosynthesis and tomography, all the development for tomographic reconstruction is applicable to tomosynthesis acquisition data. New methods have been developed since the reconstruction is now according to the x-y planes instead of x-z. However, these methods are still based on iterative algorithms such as SART and OSEM that will be described in the section 5 . To recover an $\mathrm{x}-\mathrm{y}$ plane for a specific depth an algorithm is used, superposing and shifting all the acquired projections with the subsequent point-by-point superposition of all the projections. This operation delivers a single focal plane. In order to obtain all Z-slices, it is necessary to shift the projections before performing the superposition. The shifting step determines the focal depth.

\subsection{Time of Flight (TOF)}

THz pulsed imaging has the unique feature of providing a 3D "map" of the object by using the time-of-flight of the reflected $\mathrm{THz}$ pulses [143]. In brief, a $\mathrm{THz}$ pulse is directed at the sample and the reflected beam is measured in amplitude and phase. The temporal situation of the

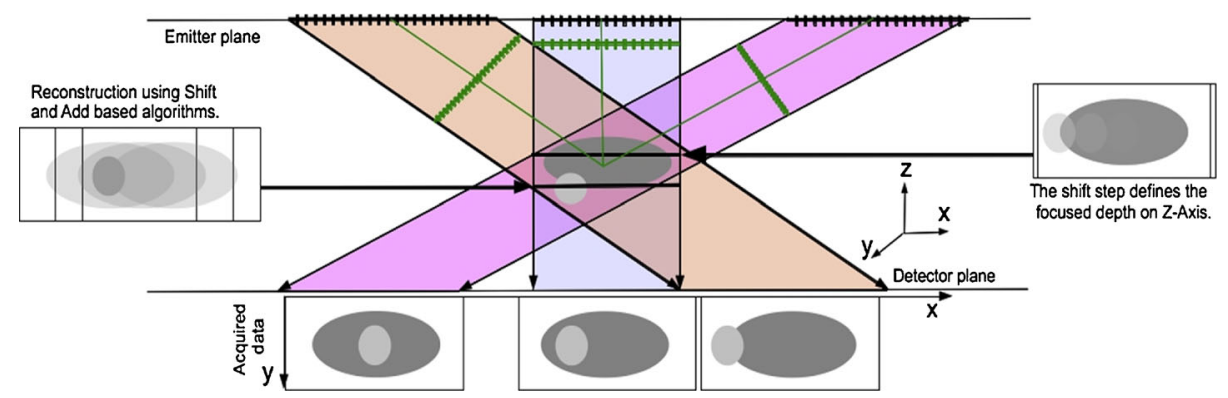

Figure 7 basics of the tomosynthesis acquisition and reconstruction process. 
reflected pulses directly indicates the presence of the interfaces along the propagation direction of the beam. In this way, by using the difference of time-of-flight from pixel to pixel, depth information of the 3D profiles of the target can be deduced. Mittleman et al [130] were the first to report on such 3D THz imaging. The 3D image of a floppy disk was produced by using time domain $\mathrm{THz}$ setup in reflection configuration. However, this new $\mathrm{THz}$ tomography tool relied on three hypotheses:

(1) Targets have no or small dispersion and diffraction properties,

(2) Reflection is weak so that multi-reflection can be ignored or suppressed [144],

(3) Refractive index is uniform within each layer.

After this first demonstration, this technique was widely adopted by various research groups. 3D imaging of many opaque materials, like metallic coins, razor blade, SD card and pharmaceutical tablets were successfully carried out by using pulsed $\mathrm{THz}$ radiation. The technique was also used to identify the presence or absence of defects in foam materials. Pre-built defects in foam insulation of space shuttle fuel tanks were detected using $\mathrm{THz}$ tomography [20]. A tomography system based on reflective THz-TDS and 2D electro-optic sampling using a high speed complementary metal-oxide semiconductor (CMOS) camera was demonstrated. The inner structures of layered samples [145] (stacked Si and Vinyl chloride plates ) were identified with 3D volume visualization within 3 minutes [146]. In this technique, as the arrival time of the $\mathrm{THz}$ waveforms can be determined with a femtosecond precision, the position of reflecting surfaces within the test object, can be resolved with accuracy of a several micrometers (provided consecutive reflections are resolved in time) [147]. For example, a very high depth resolution on the order of $1 \mu \mathrm{m}$ has been demonstrated [148] (Fig.8).

TOF THz resolution depends on the width of the incident THz pulse, and is improved when the pulse is short. When the THz pulse is not ideal (e.g., a top-hat function), but varies with time in the same way, distinction of the reflected pulse becomes difficult, and ghost interfaces

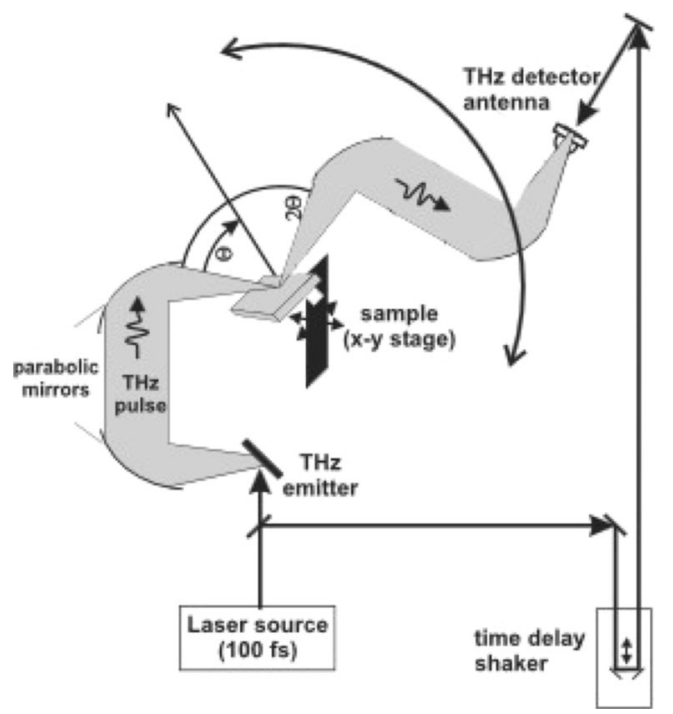

Figure 8 Principle of TOF THz tomography. Schematic taken from [149] 
can appear in the tomographic image. These ghost images can be eliminated using signal processing, for example by employing deconvolution algorithms.

The main application fields of reflection imaging concern materials where transmission measurement is prohibited and opaque materials (biological media due to the high liquid water content) which cannot be imaged in transmission mode [20]. In all the case, the very large bandwidth is a supplementary benefit, since we can access different wavelengths from each layer and then not only extract depth information but also construct a quasi three dimensional image of an object

TOF tomography is well adapted for layered targets with well-defined boundaries. However when the inner part of the sample has a more complicated constitution (internal or external shape), the multiple reflections and refractions of the $\mathrm{THz}$ radiations superpose and scatter. Yet, this analysis is possible in some cases, and resolution of sub-wavelength features $(\lambda / 10)$ has been demonstrated [150]. As a consequence, back-reflected signals are very complicated to analyze. Analysis is possible in some cases and resolution of feature of subwavelength $(\lambda / 10)$ has been demonstrated [150]. For other cases, one solution is to perform a $\mathrm{THz} \mathrm{CT}$ analysis with a complete set of projection images. Moreover, a powerful new imaging modality for terahertz radiation has been proposed [151]: T-ray reflection computed tomography. In this method, edge maps of a sample's cross-section could be computed from different reflection measurements at different viewing angles [152].

\subsection{D Holography}

3D THz holography originates from radar and optical holography technology [153]. A basic experimental configuration is presented in fig.9. The main hypothesis is that the separations between scattering centers are much larger than the wave pulse width. If it is possible to differentiate each of the multiple scattered $\mathrm{THz}$ waves of different scattering orders, a holography approach could be implemented with a terahertz beam [132]. To produce terahertz holograms, intensity and phase distributions must be measured. The interference pattern produced by the sample and reference waves is recorded and contained much more information than a focused image. Then the reconstruction of these holograms based on Fourier optics theory enables the observer to view a true three-dimensional image [154]. Ruffin et al. have demonstrated time-reversal imaging that can be considered as 2D holographic imaging in the

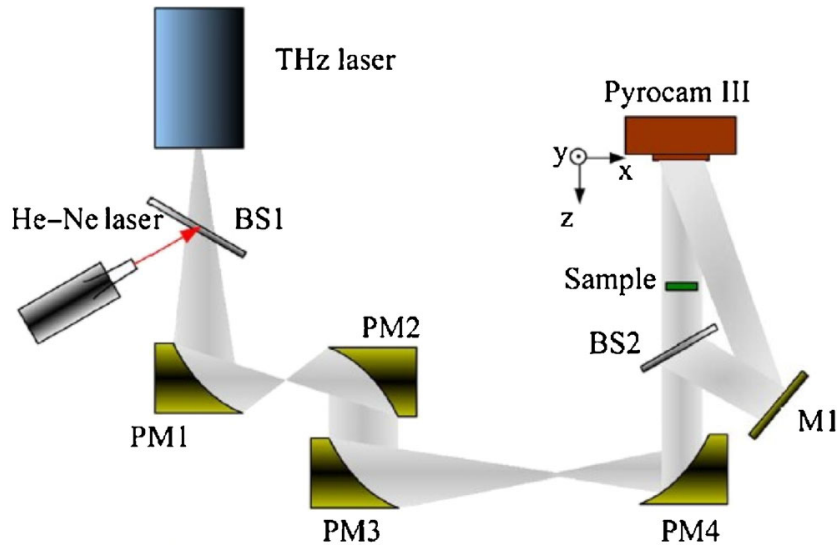

Figure 9 Experimental setup for off axis terahertz hologram recording taken from [158] 


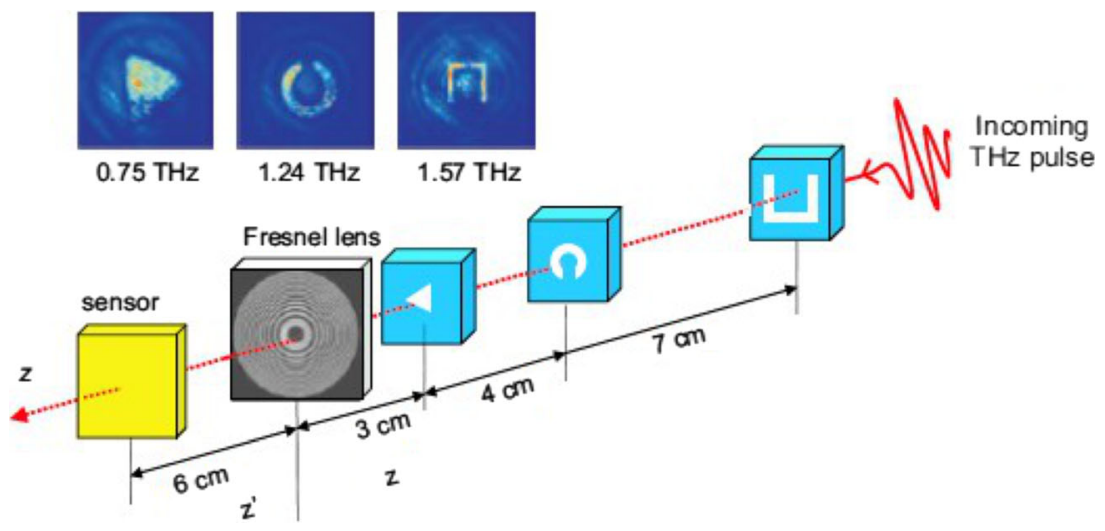

Figure 10 Schematic illustration of tomographic imaging with Fresnel lens extracted from [163]

time-domain [155]. Millimeter Wave [156, 157] or Terahertz holography [158] provides high fidelity images for targets consisting of scattered points located at well-separated planes [159]. However it has a number of pertinent deficiencies. Its extension to more complex targets is far from trivial, and in any case it does not provide accurate refractive index data on the reconstructed target. This technology which relies on measuring multiple scattering and diffraction effects, strongly affects the image quality [160] with a usually poor signal to noise ratio (SNR) owing to the high order scattering measurement [161]. As the image of the $\mathrm{THz}$ holography relies on the incident pulse, THz 3D holography does not enable one to extract spectroscopic information about the target.

\subsection{Fresnel Lenses}

Diffractive Fresnel zone plates, or Fresnel lenses may be used to focus light in place of traditional refractive lenses [162]. Fresnel lenses often have size and weight advantages over refractive lenses. However they are generally favored for narrowband applications due to their frequency dependent focal length. Wang and Zhang [163] demonstrated that this frequencydependence may be used to perform tomographic THz imaging. Using a Fresnel lens, and considering the image formed by radiation at each different frequency, we can image different samples localized at various positions along the beam propagation path onto a given imaging plane. Figure 10 is a schematic setup of Fresnel lens imaging. This distinctive property could be used to perform tomographic imaging of a target when used with broadband illumination $[164,165]$. The depth of focus of the THz wave mostly determines the resolution of this technique in the $\mathrm{z}$ dimension, so there is no restriction for narrowband imaging systems. This type of tomographic measurement is suitable if the rotation of the sample is not possible.

\subsection{Synthetic Aperture Processing (SA)}

In conventional imaging, a set of optics is used to focus a beam into a narrow beam, reaching its minimum beam dimensions in the so-called beam spot. The resolution of the system is then optimum in the center and degrades in its vicinities. Usually the Rayleigh length is considered as a reference dimension for the maximum sample thickness. As a consequence, the sample or the area to be measured must be placed where the beam spot lies. Moreover, the beam must be ideally normal to the surface of the sample to maximize signal detection. A sample whose 


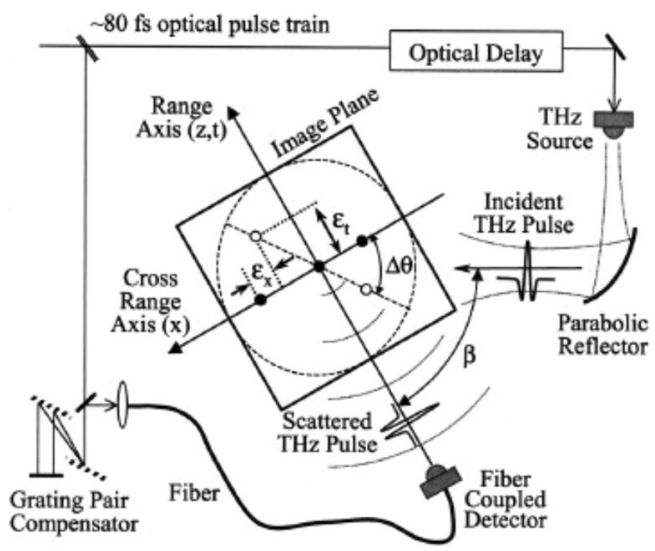

Figure 11 schematic setup for Synthetic Aperture Radar tomographic imaging [166].

thickness is comparable to or greater than the Rayleigh length will not be correctly or fully imaged in depth. In those cases, SA measurements can be of great interest $[166,167]$.

In contrast to the point-to-point measurement scheme in conventional imaging systems, Synthetic aperture systems use a diverging or unfocused beam to collect data [168]. A given target in a measurement scene can be illuminated by the sensor from several adjacent scanning positions, given the wide beam used. The collected energy from these positions will be individually much lower than in conventional imaging. Therefore a coherent integration of the received signals is applied which will significantly increase the signal to noise ratio (SNR) of the resulting image for that same target. This energy compaction process is known as Synthetic Aperture processing and is typically performed using time- or frequency-domain algorithms, yielding the former exact but computationally still very demanding results. Since these algorithms are based on the integration of matched filter and coherent raw data, the lack of correlation between noise and signals of interest is exploited; greatly reducing the influence of noise in the final reconstructed SA image.

Synthetic aperture imagery provides a constant, range-independent lateral or cross-range resolution, which is especially useful when thick samples need to be measured. The maximum thickness the system is able to measure is no longer limited by the Rayleigh length but by the sensor (operation parameters) and the sample under inspection [169]. A wide-beam approach can also be used to overcome situations such as complex scanning paths or small scanning curvatures limiting the mechanical operability of the scanner. There are several successful demonstrations of terahertz imaging with enhanced spatial resolution by a synthetic phased array. From these we extracted the typical setup shown in fig 11 [166, 170, 171].

\subsection{Time Reversal Approach}

Time reversal imaging is an innovative indirect imaging method demonstrated with pulsed $\mathrm{THz}$ radiation by Ruffin et al [87]. By exploiting the time-reversal symmetry of Maxwell's wave equations, they derived an image reconstruction algorithm based on the time-domain Huygens-Fresnel diffraction equation. This method allowed them to reconstruct 1D, 2D and 3D [172] amplitude and phase contrast objects based on the measurement of the diffracted $\mathrm{THz}$ field at multiple angles [173]. 
The resolution is given by the spatial separation of two points on the object plane that give rise to $\mathrm{THz}$ pulses with an observable timing difference at the detector. Using this method a resolution of $674 \mu \mathrm{m}$ was demonstrated, which was significantly smaller than the average wavelength of the $\mathrm{THz}$ source used. Time reversal $\mathrm{THz}$ imaging was also demonstrated for phase contrast targets and for reflection mode imaging [87]. New approaches to improve the resolution of time-reversal based $\mathrm{THz}$ imaging systems have been proposed such as the increase of the system numerical aperture via a waveguide technique adapted from ultrasound imaging and a new model-based reconstruction algorithm [174].

\subsection{THz Computed Tomography (CT)}

X-Ray computed tomography was originally developed as a medical imaging procedure that produced tomographic images or 'slices' of specific patient areas. These cross-sectional images are helpful for diagnostic and therapeutic purposes in various medical disciplines. A real threedimensional image of the inner part of an object is reconstructed from a series of twodimensional X-ray images taken around a single axis of rotation (the emitter and sensors spin around the patient). X-ray CT is now used for industrial applications and many companies are equipped with X-ray tomographic systems devoted to quality control, defect localization etc. However, the dangerous effects of X-ray strongly reduce its applications.

Terahertz CT was introduced by Fergusson et al. in 2002 [138]. Terahertz computed tomography presents new potential because we can measure amplitude as well as spectral phase information in comparison with X-ray imaging. With Terahertz CT, it's not utopist to identify or compare different substances and localize them in a nondestructive manner. A typical experimental configuration is presented in Fig 12. Brahm et al. also demonstrated recently a spectral analysis of materials using THz CT [175]. Lactose and glucose inserted in a polystyrene block have been separated due to strong absorption lines present in their terahertz spectra. Nevertheless, the main fundamental limitation that slows down the transfer out of the laboratories is the absorption phenomena that limit the thickness of the sample to be imaged. For a potential application, a long phase of feasibility must be envisaged because many experiments must be conducted with real samples and some tradeoffs are such as the optimal wavelength to combine transparency, resolution, and ability to fairly image the sample. The long acquisition time arising from use of $\mathrm{THz}$ time-domain spectroscopy has been overcome recently [176]. Nevertheless the technological development presented seems difficult to transfer because of the complexity and the cost of high power optical and terahertz sources. Another limitation comes from the sample itself for which the transparency must be sufficient in the terahertz bandwidth [177]. This new approach requires new algorithms and

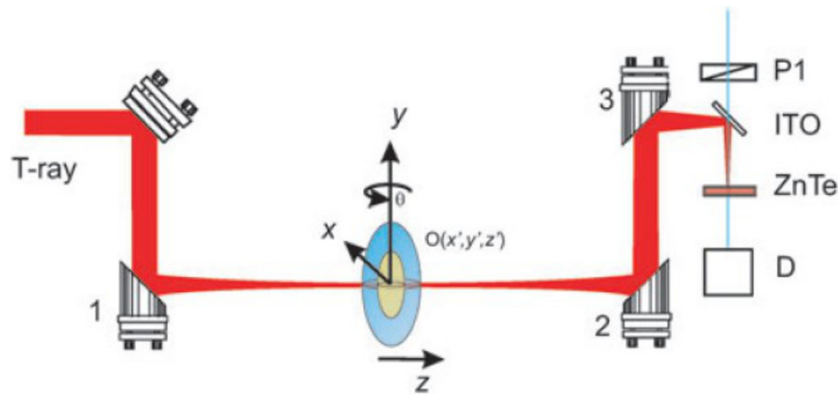

Figure 12 setup of $\mathrm{THz} \mathrm{CT}$ experiment [132] 
reconstruction strategies because terahertz beam propagation is far from that for X-rays and all the existing processing techniques must be revisited.

\section{Data Processing and Reconstruction}

Tomography is an imaging technique used to reconstruct the volume of an object from a set of projections coming from the exterior of the sample under investigation. This technique has been known since the beginning of the $20^{\text {th }}$ century with Johann Radon's work $[178,179]$. But the first tomographic acquisition system (X-Ray Computed Tomography scan) was developed by G.N Hounsfield and A. Cormark in the 1970's [180]. It is composed of an X-Ray transmitter diametrically opposed to a set of sensors. The patient is subjected to X-Rays from different points of view giving a series of measures corresponding to the ray attenuation in the traversed tissues. The data are then treated by a computer to reconstruct slice views or 3Dviews.

Since X-ray CT scan development, several other acquisition systems have been developed, such as Positron Emission Tomography (PET) [181, 182], Single Photon Emission Computed Tomography (SPECT), Transmission Electron Microscopy (TEM) and electro-magnetic radiation in the continuous or pulsed Terahertz frequencies (THz CT) $[138,183]$. Even if the properties, the physical medium and the data revealed by the reconstructed images differ between each acquisition system, they are all based on the measurement of the attenuation of an X-ray going through matter.

Mathematically, the tomographic process is composed of two distinct steps. The first one describes the acquisition (direct model), i.e. how to get the measure set from the physical phenomenon (for instance, the X-Ray attenuation). The inverse model defines the manner to reconstruct the volume from the acquired set. Since these models are defined in the continuous domain, they are not applicable directly and they have to be discretized. Moreover, the acquisition process is not perfect and the acquired data will be affected by static and dynamic misalignments. Noise during the acquisition and approximations induced by the discretization generate errors in the reconstructed data. Consequently, one of the research areas in tomography in recent times has been to develop methods to reduce the discretization and noise errors in the reconstructed images.

In this part, we will explore the typical reconstruction methods (and their major optimizations) to obtain an image of observed objects. First, the direct methods are developed; we define the acquisition and reconstruction models, in the spatial domain with the Radon transform and in the frequency domain with the Fourier Slice Theorem [184]. Second, we detail another kind of reconstruction: iterative methods. In particular, we explain the Karcmarz method giving well-known algebraic reconstructions such as the Simultaneous Algebraic Reconstruction Technique SART [185] and we develop methods based on stochastic process such as the Expectation Maximization (EM) [186].

\subsection{Direct Methods}

We develop in this section the acquisition model based on the Radon transform. The inverse model giving the representation of the acquired object is detailed through the back projection of filtered projections. Direct reconstruction in the frequency domain is also explained. These models are defined in the continuous domain and have to be discretized to be applied in tomography. The discretization step, its limitations and main goals developed to minimize limitation effects, are the main drawbacks of these approaches. 


\section{a. From the physical phenomenon to the reconstruction}

The rays used in tomography (for instance X-Rays, THz radiation, electrons) have sufficient penetration power to enable them to pass through the sample. During this propagation, the beam encounters attenuation depending on the density and composition of the material. It is for instance the basic principle for X-Ray radiography. After the acquisition of 2D projection data, known as a sinogram, a mathematical calculus named retro-projection can reconstruct the 2D slice of the object [187, 188].

Let's consider the following example with a $2 \mathrm{D}$ centered orthonormal domain (on the left on Fig.13). Each $1 \mathrm{D}$ projection $R_{\theta}$ is defined for an angle $\theta$. A projection line along $\theta$ depends on its position given by a module $\rho$. Such a line is denoted $(\theta, \rho)$. The measured value depends on data obtained by in the $\theta$ direction and corresponds to the attenuation undergone by the rays into the matter along this line.

For a given angle $\theta$, a set of modules and their values define the $\theta$-projection, denoted $R_{\theta}$ (Fig.13a.). We define a sinogram, denoted $S$, as the set of projections acquired around the object during a complete rotation of the sample. The sinogram values are used to reconstruct the acquired domain with back-projection. On the scheme on the right (Fig.13.b), the projection is back-projected into the domain to reconstruct. One projection is not sufficient to recover the original domain (compare the grey zone in Fig 13a and b). If the sinogram contains several projections (Fig.14a.), the original domain is reconstructed more accurately (Fig.14b.).

\section{b. Radon Transform}

The acquisition/reconstruction process is modeled by the Radon transform, defined by Johann Radon in 1919. The direct transform $R$ is a projection line acquisition. It transforms a 2D function defined by $f(x, y)$ into a 1D projection $[178,179]$. It is given by the following formula:

$$
R_{\theta}(\rho)=\int_{-\infty}^{\infty} \int_{-\infty}^{\infty} f(x, y) \delta(\rho-x \cos \theta-y \sin \theta) d x d y
$$

Where $\theta$ and $\rho$ are the angular and radial coordinates of the projection line $(\theta, \rho)$ respectively, and $\delta(\cdot)$ is the Dirac impulse. So, $R_{\theta}(\rho)$ is the absorption sum of all points traversed by
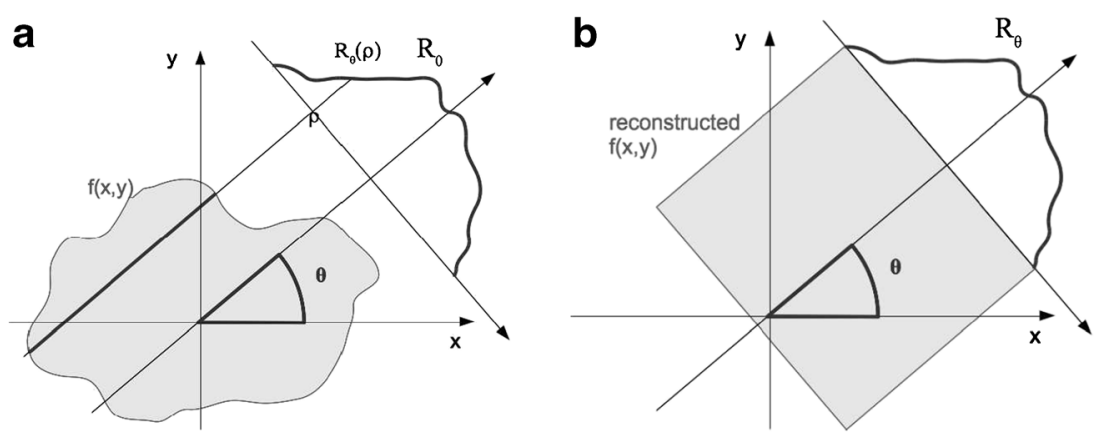

Figure 13 (a) A projection line is defined by an angle $\theta$ and a module $\rho$. Its value depends on traversed $f(x, y)$ values and corresponds to the attenuation undergone by the rays along the black line. (b) One projection is not sufficient to recover the original function. 

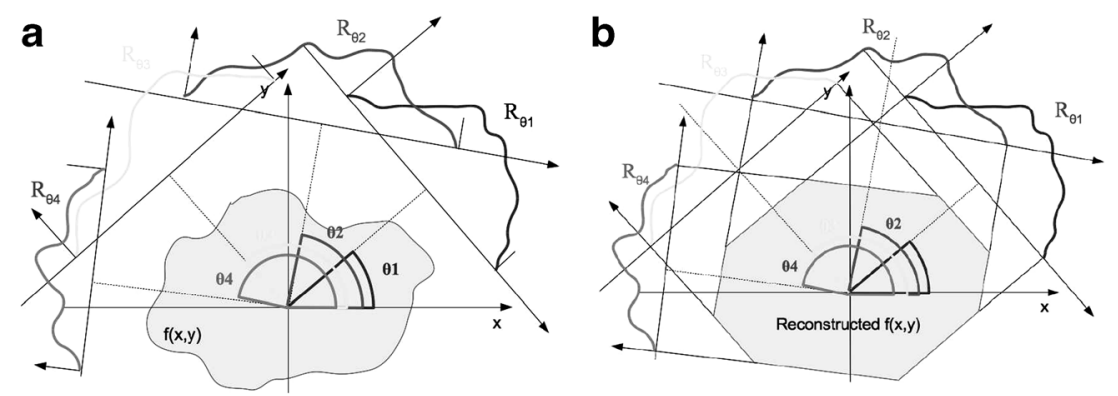

Figure 14 (a) Acquisition along several angles. (b) The intersection of data contained on the different projections allows reconstructing the original function more accurately.

the line in the domain. The inverse reconstruction recovers the original function $f$ from the projections. Supposing a sinogram $S$ with infinitely many projection values such that $\theta \in[0, \pi]$ and $\rho \in \mathbb{R}$, the inversion is given as :

$$
\mathrm{f}(\mathrm{x}, \mathrm{y})=\int_{0}^{\pi} \int_{-\infty}^{+\infty} \mathrm{F}(|\rho|) \mathrm{R}_{\theta}(\rho) \delta(\rho-\mathrm{x} \cos \theta-\mathrm{y} \sin \theta) \mathrm{d} \rho \mathrm{d} \theta
$$

Where $F$ is the Fourier transform. First, this inversion applies a ramp filter on each projection to increase details. Second, the point $(x, y)$ value is computed from the sum of the filtered projections. This method is called Filtered Back-Projection (FBP).

\section{c. Fourier Slice Theorem}

Another approach consists of inverting the 2D Fourier transform of the original function by combining the information contained in the projection Fourier transforms. The Fourier Slice Theorem (FST) specifies that the 1D Fourier transform of a projection $R_{\theta}$ (denoted $F_{1 D} R_{\theta}$ ) of $f(x, y)$ along an angle $\theta$ corresponds to a line of the Fourier space (denoted $F_{2 D}(X, Y)$ ) of $f$ along the same angle $\theta$. Indeed, expressing the function $f$ in polar coordinates, with $x=r \cos \varphi$ and $y=r \sin \varphi$, equation (1) becomes:

$$
\mathrm{R}_{\theta}(\rho)=\int_{0}^{2 \pi} \int_{-\infty}^{\infty} \mathrm{f}(\mathrm{r} \cos \phi, \operatorname{rsin} \phi) \delta(\rho-\mathrm{r} \cos (\varphi-\theta))|\mathrm{r}| \mathrm{dr} \mathrm{d} \varphi
$$

The Fourier transform $F_{1 D}$ of the projection $R_{\theta}(\rho)$ is:

$$
F_{\theta}(\nu)=F_{1 D}\left(R_{\theta}(\rho)\right)=\int_{-\infty}^{\infty} R_{\theta}(\rho) \mathrm{e}^{-2 \mathrm{i} \pi \rho v} \mathrm{~d} \rho
$$

Combined with (3) this becomes:

$$
F_{\theta}(\nu)=\int_{0}^{2 \pi} \iint \mathrm{f}(\mathrm{r} \cos \phi, \operatorname{rsin} \phi) \delta(\rho-\operatorname{rcos}(\varphi-\theta))|\mathrm{r}| \mathrm{e}^{-2 \mathrm{i} \pi \rho \nu} \mathrm{d} \rho \mathrm{drd} \varphi
$$

Since the Dirac impulse is not null when $\rho=\operatorname{rcos}(\varphi-\theta)$, this simplifies to:

$$
F_{\theta}(\nu)=\int_{0}^{2 \pi} \int_{-\infty}^{\infty} \mathrm{f}(\mathrm{r} \cos \phi, \mathrm{rsin} \phi)|\mathrm{r}| \mathrm{e}^{-2 \mathrm{i} \pi \rho v \cos (\varphi-\theta)} \mathrm{drd} \varphi
$$


Consider the 2D Fourier Transform $\overline{\mathrm{F}}_{2 D}(\psi, \nu)$ of $f$ in polar coordinates, with $X=\boldsymbol{v} \cos \psi$ and $Y=v \sin \psi$ :

$$
\overline{\mathrm{F}}_{2 D}(\psi, \nu)=\int_{\theta}^{2 \pi} \int_{-\infty}^{\infty} \mathrm{f}(\mathrm{r} \cos \phi, \operatorname{rsin} \phi)|\mathrm{r}| \mathrm{e}^{-2 \mathrm{i} \pi \rho v \cos (\varphi-\theta)} \mathrm{drd} \varphi
$$

From equations (6) and (7), we obtain the equation (8) corresponding to the FST [184]:

$$
\mathrm{F}_{\theta}(\nu)=\mathrm{F}_{1 D}\left(\mathrm{R}_{\theta}(\rho)\right)=\mathrm{F}_{2 D}\left(f(r \cos (\phi), r \sin (\varphi))=\overline{\mathrm{F}}_{2 D}(\psi, \nu)_{\psi=0}\right.
$$

So, we can populate the 2D Fourier transform of the original function from the projection Fourier transform data. The original function is then obtained by an inverse 2D Fourier Transform.

\section{d. Acquisition properties}

Contrary to an ideal acquisition, a real-condition acquisition is composed by a finite number of projections $\mathrm{N}_{\theta}$ that are typically uniformly distributed between 0 and $\pi$. The sample number denoted $\mathrm{N}_{\rho}$ is also finite and remains constant during acquisition. The angular step between two successive projections is $d \theta=\frac{\pi}{N_{\theta}}$. Similarly, we denote $d \rho$ the sampling step on the projections, i.e. the distance between two successive projection lines. During the discrete image reconstruction $I$, sized $W \times H$ pixels, the sampling ratio between projections and image is usually $d \rho=\frac{M A X(W H)}{N \rho}=1$.

An acquisition with $\mathrm{N}_{\theta}$ projections composed of samples gives the sinogram $\mathrm{S}^{*}$, which is a sub-set of the ideal sinogram $S$. It is represented by a $2 \mathrm{D}$ image, sized $\mathrm{N}_{\theta}$ $\mathrm{x} \mathrm{N}_{\rho}$, where each line corresponds to the acquisition values of one projection. Supposing that $i_{\theta}$ and $i_{\rho}$ are the projection and module indices such as $0 \leq \mathrm{i}_{\theta}<\mathrm{N}_{\theta}$ and $0 \leq \mathrm{i}_{\rho}<\mathrm{N}_{\rho}$, the pixel $\left(\mathrm{i}_{\theta}, \mathrm{i}_{\rho}\right)$ contains the value,$R_{\theta}(\rho)$.

Let us consider a continuous model representing the Shepp-Logan phantom [184]. This model is based on an analytic definition of the objects defined in [178]. Fig.15(a) represents this domain in an image sized $512 \times 512$. An acquisition is done following 180 angles uniformly distributed between 0 and $\pi$, with 512 samples per projection. The resulting sinogram is given on Fig.15(b). The first line represents the 512 values of the $0^{\circ}$-projection, the second line contains the values of the $1^{\circ}$ projection and so on.
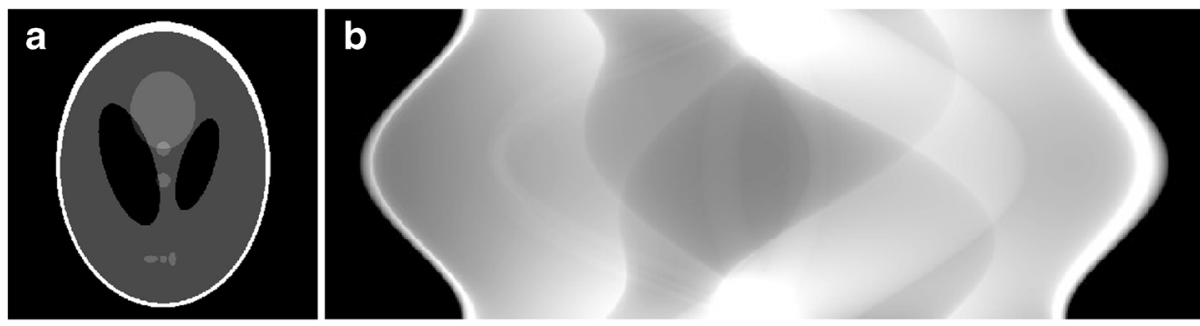

Figure 15 (a) Continuous Shepp-Logan phantom represented in an image sized 512×512. (b) Acquired sinogram following 180 projections of 512 samples. 
Such a sinogram can be used to reconstruct an image $I$ representing the acquired function. This one is performed using discrete versions of the Radon inversion or Fourier reconstruction. The discrete inverse Radon transform, denoted $R^{-1}$, reconstructs an image $I$ from an acquisition using the following formula:

$$
R^{-1}(i, j)=\mathrm{I}(\mathrm{i}, \mathrm{j})=\sum_{i_{\theta}=0}^{N_{\theta}-1} \sum_{i_{\rho}=0}^{N \rho-1} R_{\theta}^{B F P}(\rho) \rho k(\theta, \rho, i, j)
$$

Where $p k$ is a pixel kernel used to determine the manner that the projection line crosses the pixel and $R_{\theta}^{B F P}(\rho)$ is the filtered projection value given by:

$$
R_{\theta}^{B F P}(\rho)=\sum_{\rho s}|\nu|\left(\sum_{\nu} \mathrm{R}_{\theta}\left(\rho_{s}\right) e^{-i 2 \pi \rho_{s} \nu}\right) e^{i 2 \pi \rho \nu}
$$

For Radon inversion, we have to discretize the Fourier reconstruction to get an image from projection Fourier transforms. If we consider this theorem in a discrete point of view, the simplification made in Eq.(5) is impossible because each polar point is not exactly localized in the center of a pixel [178]. Consequently:

$$
\mathrm{F}_{\theta}(\nu)=\overline{\mathrm{F}}_{2 D}(\psi, v)_{\psi=0}=\sum_{\phi=0}^{N_{\theta}} \sum_{\rho=0}^{N_{\rho}}\left[\sum_{r=0}^{N_{\rho}} I(r \cos \varphi, r \sin \phi)|r| \mathrm{e}^{-2 \mathrm{i} \pi \rho \cos (\phi-\theta)}\right] \Delta(\rho-r \cos (\phi-\theta)
$$

Sum between brackets corresponds to the 1D Fourier transform. The double-sum outside the brackets determines the polar point(s) used to compute the pixel $\overline{\mathrm{F}}_{2 D}(\psi, v)$. Then, this discretization is an interpolation from the polar grid containing Fourier projection points to a Cartesian grid corresponding to the image Fourier space. These discretization issues are widely treated in the literature concerning computed tomography [189-191] and their typical defects are illustrated in fig.16.

\subsection{Iterative Reconstructions}

Contrary to direct methods, iterative processes can correct the error induced by one (or a set of) projection(s) according to the state of the image under reconstruction and the data available on the other projections. First, algebraic techniques are detailed. Afterwards, we introduce methods based on stochastic process.
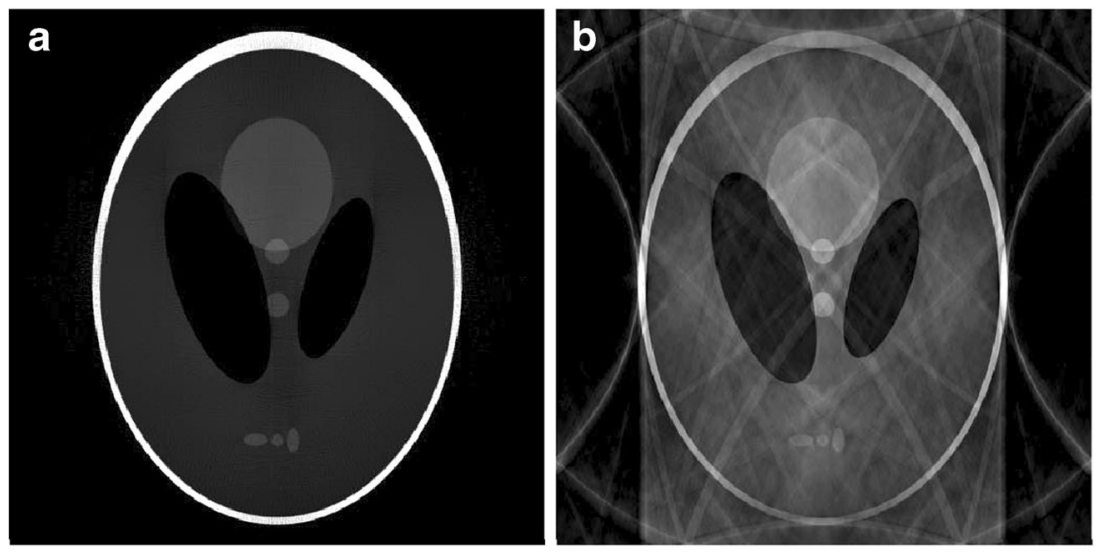

Figure 16 (a) slice of Sheep logan model (b) Reconstruction using Fourier inversion from sinogram Fig.15(b). 


\section{a. Algebraic Methods}

Algebraic methods, which are iterative, were introduced in the 1970's by Gordon et al [192, 193] as the Algebraic Reconstruction Technique (ART). Based on the Karzmarz theorem, this iterative algorithm approaches the linear equation system solution through an update of each pixel value. The pixel correction is done according to the error measured between the initial sinogram and the sinogram recomputed from the image at the previous iteration. Considering the discrete geometry, the projection line value is a linear combination of pixel $(i, j)$ values weighted by a pixel kernel $p k$. It is defined by:

$$
R_{\theta}(\rho)=\sum_{i=0}^{W-1} \sum_{j=0}^{H-1} \rho k(\theta, \rho, i, j) I(i, j)
$$

The sinogram $R$ given by a vector sized $\mathrm{N}_{\theta} \mathrm{xN}_{\rho}$ and the image to reconstruct $I$ is defined by a vector sized $W \times H$. The weight matrix $A$ is defined with $\mathrm{N}_{\theta} \mathrm{xN}_{\rho}$ lines and $W \times H$ columns. The following linear equation system gives the overall pixel contributions in the projection lines:

$$
R=A I
$$

Inversion of this system recovers the original vector $I$ from the projection data $R$ :

$$
\mathrm{I}=\mathrm{A}^{\mathrm{T}} \mathrm{R}
$$

Equation system resolution is the main goal of the algebraic techniques. Knowing the image and acquisition sizes, this system is too large to be solved using typical matrix inversion techniques. Moreover, linear approximations and the projection noise do not assume an exact resolution. The Karzmarz method approaches the solution by a successive estimation of the vector $\mathrm{I}^{\mathrm{k}}$ at the iteration $k$. Each pixel $\mathrm{I}^{\mathrm{k}}(\mathrm{i}, \mathrm{j})$ is updated by comparing the measured value $R_{\theta}(\rho)$ with the value $R_{\theta}^{k}(\rho)$ computed from $\mathrm{I}^{\mathrm{k}-1}$ (using equation 9). Iteratively, the result converges to a solution minimizing the error between $R$ and $\mathrm{R}^{\mathrm{k}}$ and approaching the ideal solution $I$. This method needs an initial image which can be a direct method result. However, the solution can suffer from artifacts given by this initial result [194]. Usually, an uniform image [195] where each pixel is valued with the initial sinogram average value is chosen.

Several variations of the ART method have been developed, such as:

- Multiplicative ART (MART), where the update is computed according to the ratio between $R$ and $\mathrm{R}^{\mathrm{k}}[196]$;

- Adaptive ART (AART) uses an adaptive adjustment of a relaxation parameter at each reconstruction step [197];

- Simultaneous Iterative Reconstruction Technique (SIRT) [198], where each pixel is directly updated from all the projection lines (of all the projections) at once;

- Simultaneous ART (SART) is a compromise between ART (projections are independent and an optimized access scheme is used) and SIRT (update from several projection lines). It uses all the projection lines from one and only one projection to update the image. The pixel error is then averaged by the use of several values (at once) in the sinogram and the update is optimized by a projection access scheme. 


\section{b. Introduction to Stochastic Methods}

Methods based on statistic interpretations formalize the reconstruction problem as follows: What is the most probable image I knowing the observed $R$ [186]. Resulting iterative algorithms try to maximize the probability $p(I \mid R)$, i.e. the probability to get $I$ according to the projections $R$. Bayes theorem defines:

$$
p(I \mid R)=p(R \mid I) \times p(\mathrm{I}) / \mathrm{p}(\mathrm{R})
$$

$p(I / R)$ is the value to optimize; $p(R \mid I)$ is the probability to have the projections $R$ according to the image $I$. This one is the likelihood of the projections. $p(I)$ is the a priori of the image $I$ and $p(R)$ is the projection a priori. $p(R)=1$ because the projections are known. Restricting the solution space $p(I)=1$ :

$$
p(I \mid R)=p(R \mid I)
$$

To maximize the probability to get the image $I$ knowing the projections $R$, we have to maximize the projection likelihood knowing an image $I$. This maximization is given by minimizing the distance between calculated projections and observed projections. The process is then similar to the algebraic methods because this minimization is done by iterative updates (in $k$ ) of $I$. The Maximum Likelihood Expectation Maximization (MLEM) [186] defines the likelihood $p(R \mid I)$ by modeling $\mathrm{R}^{\mathrm{k}}$ as a Poisson distribution and we can use a logarithmic version to exploit this model.

The goal is to maximize a mathematical expression and this maximum is obtained when the partial derivative along $I$ is null. Maximization of the likelihood consists of minimization of its partial. Since each pixel value is independent, we can consider each pixel independently to globally minimize the partial derivate. Then, the first part of MLEM algorithm computes the expectation of the likelihood according to the set of projections of the image under reconstruction. The second step computes the expectation maximization by canceling the partial derivatives according to $I$. This maximization is done by the iterative pixel update. Because the update is multiplicative and the maximization takes into account the projection set, the MLEM method is equivalent to a simultaneous MART method. Then, it is sometimes denoted SMIRT (Simultaneous Multiplicative Iterative Reconstruction Technique). Another probabilistic model based on a Gaussian distribution has also been proposed. It leads to the widespread conjugate gradient methods [199] which are similar to the MLEM in additive form [200]. Due to slow convergence of the MLEM method, several optimizations based on separating the projection sets in several ordered ones has been proposed. This ordered subset technique is now commonly used for its high quality accuracy and convergence speed in medical fields. For THz tomography, a quantitative comparison of different method has been studied [183].

\subsection{Propagation Beam Examination and Modeling}

In the X-ray CT transmission process, the beam is supposed constant due to its short wavelength and the very low attenuation. For the same reason, the energy distribution is supposed uniform on a cross-section disk. This specificity allows the Radon model to be employed directly to implement reconstruction objects. In $\mathrm{THz} \mathrm{CT}$ imaging, the propagation beam follows a Gaussian distribution, which is determined by both the THz wave properties and the lenses used to focus or the shape the beam. The radius of the Gaussian beam from the 
beam axis takes the minimum value $w_{0}$ at the so- called beam waist. According to the wavelength $\lambda$, the radius at the position $z$ from the beam waist is:

$$
w(z)=w_{0} \sqrt{1-\left(\frac{z}{z r}\right)^{2}}
$$

Where $z_{r}=\frac{\pi w_{O}^{2}}{\lambda}$ is the Rayleigh range. Moreover, the energy distribution at the crosssection is given by:

$$
E(r, z)=E_{0}\left(\frac{w_{0}}{w(z)}\right)^{2} e^{\frac{-2 r^{2}}{w^{2}(z)}}
$$

where:

- $\mathrm{r}$ is the distance from the beam axis,

- $E_{0}$ is the energy at the center of the beam waist.

Note that the energy decreases from the center to the edge of the beam following a Gaussian distribution. A profile at the central plane of the beam observed for a $240 \mathrm{GHz}$ is given in Fig.17. It shows the variation of the radius (horizontally) and the Gaussian distribution of the energy (vertically).

Knowing the source properties and using equations (17) and (18), the observed Gaussian beam can be computed to simulate a given source. When a lens focuses the beam, the $\mathrm{THz}$ source is modified by another Gaussian propagation model corresponding to the lens impact. In such a case, the overall Gaussian beam can be expressed as a convolution of both Gaussian beams modeling the source and the lens. If lens properties are unknown, the overall model can be estimated from the beam propagation observations using a non linear least square 3D Newton-Gauss algorithm.

This Gaussian beam modeling has been successfully exploited by Recur et al as a convolution filter in the iterative techniques SART and OSEM. This considerably improves the quality and the accuracy of reconstructed images [183, 201]. This much more rigorous quantitative approach provides a new insight for the improvement of 3D THz CT imaging. That also proves that using BFP without any deconvolution leads to dramatic and erroneous images even in the simple case of a metallic bar.

Out of the green zone in the Fig.17, artifacts are present in the reconstructed object which could lead to important errors. To illustrate the efficiency of these methods, we imaged a Rohacell polymer sample which is quite transparent in the lower part of the terahertz bandwidth (from $0.1-3 \mathrm{THz}$ ). Its dimensions are $30 \times 34 \times 37 \mathrm{~mm}^{3}$ : We inserted two metallic balls as eyes and a screw in an oblique direction inside the polymer skulls. In order to perform a 3D reconstruction of Terakenstein, we first record a 2D transmission image of the sample by moving the object in the $\mathrm{X}$ and $\mathrm{Y}$ directions with a scan step of $1 \mathrm{~mm}$ in both directions. The acquisition time for a $(50 \times 50)$ pixel image size is about 20 seconds with a high speed moving

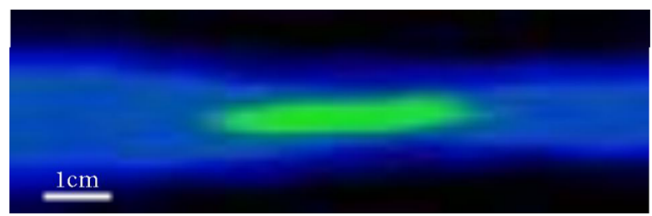

Figure 17 Propagation beam profile in the central plane from [201] 

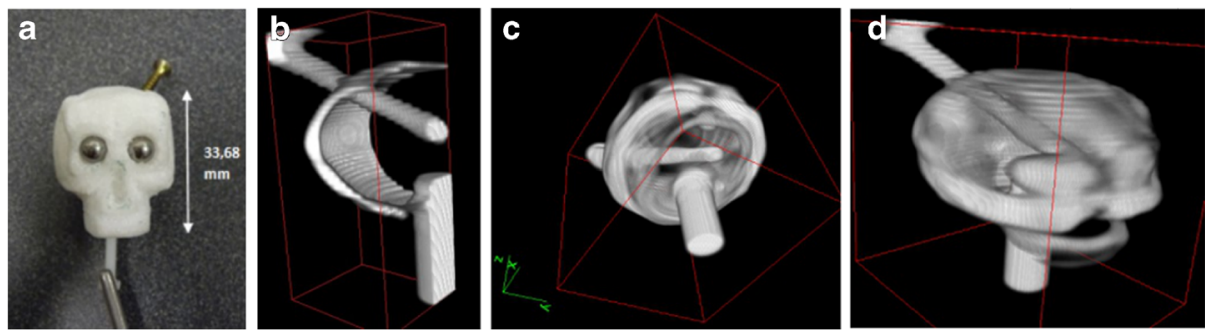

Figure 18 Reconstruction of Terakenstein: the original object (a), a detail of the metal screw inside de skull (b), a view from down-top (c) and a 3D reconstructed view (d) [201].

table. Then, the sample is rotated by a rotation step $\theta$ in order to provide a different visualization of the object. The operation is repeated $N$ times from $\theta=0 \circ$ to $180^{\circ}$ and we finally get a set of $N$ projections of the sample corresponding to the different angles of visualization. From all these projection images, we reconstruct the sinogram of the object which represents, for a given horizontal slice, the evolution of the transmitted $\mathrm{THz}$ amplitude as a function of the rotation angle. Finally we apply FBP, SART or OSEM algorithms to reconstruct the final 3D volume of the sample. During this back-projection procedure we deconvolued the volume column-by-column using the Gaussian beam filter we developed. The reconstructed views of Terakenstein are shown in the Fig.18. We can observe and measure all the details such as the metal screws inside the skull. A better accuracy and quality is found especially when using iterative techniques in comparison with the FBP algorithm.

\section{Conclusion}

The field of terahertz imaging is extremely diverse. Terahertz science and technology is a melting pot of interdisciplinary domains such as physics, chemistry, engineering and optic that makes this discipline challenging. Nowadays a lot of techniques and methods can be accessed by an increasing number of scientists. There are several reasons for the expansion of this field over the last few years. One is the access to spectroscopic responses in the terahertz range that reveals new physical information. Coupling with an imaging situation, it gives new tools and new visions of samples and allows the volume inspection due to sufficient penetration length, especially with frequencies smaller than $1 \mathrm{THz}$. This enlarges imaging applications, ranging from quality control to security screening. Several of these applications are illustrated in this paper. In many cases, terahertz imaging applications have been demonstrated to be an efficient and elegant solution. In order to gain more extensive recognition for this technology, research and communication to industrial partners are needed. Jointly a more robust and less expensive version of a terahertz imaging system will open new applications. Possible future improvements are related to semiconductor or optical laser sources and detector (Quantum Cascade laser and all fiber TDS experiment, and THz camera [202] for example). The terahertz science and especially spectro-terahertz imaging will probably be an emerging discipline and an efficient tool for a lot of industrial applications.

Acknowledgments We would like to express sincere thankfulness to each of the persons who have permitted us to display some results of their work for the review paper. We would also acknowledge financial support from the European community thanks to the contract DOTNAC (Development and Optimization of THz NDT of Aeronautics Composite multilayered structures) - Contract N 266320 / FP7-AAT-2010-RTD-1 - http://www. 
dotnac-project.eu/ and the Agence Nationale de la Recherche (ANR) for their support in the InPoSec project (www.inposec.org).

\section{References}

1. Zhang, X.C., Terahertz wave imaging: horizons and hurdles. Physics in Medicine and Biology, 2002. 47(21): p. 3667-3677.

2. Hangyo, M., M. Tani, and T. Nagashima, Terahertz time-domain spectroscopy of solids: a review. International journal of infrared and millimeter waves, 2005. 26(12): p. 1661-1690.

3. Mittleman, D.M., R.H. Jacobsen, and M.C. Nuss, T-ray imaging. IEEE Journal of Selected Topics in Quantum Electronics, 1996. 2(3): p. 679-692.

4. Cooper, K.B., et al., A high-resolution imaging radar at $580 \mathrm{GHz}$. IEEE Microwave and Wireless Components Letters, 2008. 18(1): p. 64-66.

5. Cooper, K.B., et al., Penetrating 3-D Imaging at 4-and 25-m Range Using a Submillimeter-Wave Radar. IEEE Transactions on Microwave Theory and Techniques, 2008. 56(12): p. 2771-2778.

6. Darmo, J., et al., Imaging with a Terahertz quantum cascade laser. Optics Express, 2004. 12(9): p. 18791884 .

7. Fitzgerald, A.J., et al., Catalogue of human tissue optical properties at terahertz frequencies. Journal of Biological Physics, 2003. 29(2-3): p. 123-128.

8. Fitzgerald, A.J., et al., Terahertz pulsed imaging of human breast tumors. Radiology, 2006. 239(2): p. 533540 .

9. Nakajima, S., et al., Terahertz imaging diagnostics of cancer tissues with a chemometrics technique. Applied Physics Letters, 2007. 90(4): p. 041102.

10. Watanabe, Y., et al., Component spatial pattern analysis of chemicals using terahertz spectroscopic imaging. Applied Physics Letters, 2003. 83(4): p. 800-802.

11. Beard, M.C., G.M. Turner, and C.A. Schmuttenmaer, Progress towards two-dimensional biomedical imaging with THz spectroscopy. Physics in Medicine and Biology, 2002. 47(21): p. 3841-3846.

12. Crowe, T.W., et al., Terahertz sources and detectors and their application to biological sensing. Philosophical Transactions of the Royal Society of London Series a-Mathematical Physical and Engineering Sciences, 2004. 362(1815): p. 365-374.

13. Stringer, M.R., et al., The analysis of human cortical bone by terahertz time-domain spectroscopy. Physics in Medicine and Biology, 2005. 50(14): p. 3211-3219.

14. Siegel, P.H., Terahertz technology. Microwave Theory and Techniques, IEEE Transactions on, 2002. 50(3): p. $910-928$.

15. Xu, J.Z., C.L. Zhang, and X.C. Zhang, Recent progress in terahertz science and technology. Progress in Natural Science, 2002. 12(10): p. 729-736.

16. Loeffler, T., et al., All-optoelectronic terahertz imaging systems and examples of their application. Proceedings of the IEEE, 2007. 95(8): p. 1576-1582.

17. Siebert, K.J., et al., Continuous-wave all-optoelectronic terahertz imaging. Applied Physics Letters, 2002. 80(16): p. 3003-3005.

18. Tonouchi, M., Cutting-edge terahertz technology. Nature Photonics, 2007. 1(2): p. 97-105.

19. Stoik, C.D., M.J. Bohn, and J.L. Blackshire, Nondestructive evaluation of aircraft composites using transmissive terahertz time domain spectroscopy. Optics Express, 2008. 16(21): p. 17039-17051.

20. Zhong, H., et al., Nondestructive defect identification with terahertz time-of-flight tomography. IEEE Sensors Journal, 2005. 5(2): p. 203-208.

21. Karpowicz, N., et al. Non-destructive sub-THz CW imaging. in Integrated Optoelectronic Devices 2005. 2005. International Society for Optics and Photonics.

22. Baxter, J.B. and C.A. Schmuttenmaer, Conductivity of $\mathrm{ZnO}$ nanowires, nanoparticles, and thin films using time-resolved terahertz spectroscopy. Journal of Physical Chemistry B, 2006. 110(50): p. 25229-25239.

23. Ueno, Y. and K. Ajito, Analytical terahertz spectroscopy. Analytical Sciences, 2008. 24(2): p. $185-192$.

24. Fischer, B., et al., Chemical recognition in terahertz time-domain spectroscopy and imaging. Semiconductor Science and Technology, 2005. 20(7): p. S246-S253.

25. Wietzke, S., et al., Industrial applications of THz systems. 2009: p. 738506-738506.

26. King, M.D., W.D. Buchanan, and T.M. Korter, Identification and Quantification of Polymorphism in the Pharmaceutical Compound Diclofenac Acid by Terahertz Spectroscopy and Solid-State Density Functional Theory. Analytical Chemistry, 2011. 83(10): p. 3786-3792.

27. El Haddad, J., et al., Review in terahertz spectral analysis. TrAC Trends in Analytical Chemistry, 2013. 44: p. $98-105$. 
28. Hu, B.B. and M.C. Nuss, Imaging with terahertz waves. Optics Letters, 1995. 20(16): p. 1716.

29. Jiang, Z.P., X.G. Xu, and X.C. Zhang, Improvement of terahertz imaging with a dynamic subtraction technique. Applied Optics, 2000. 39(17): p. 2982-2987.

30. Jiang, Z.P. and X.C. Zhang, Terahertz imaging via electrooptic effect. IEEE Transactions on Microwave Theory and Techniques, 1999. 47(12): p. 2644-2650.

31. Jiang, Z.P. and X.C. Zhang, 2D measurement and spatio-temporal coupling of few-cycle THz pulses. Optics Express, 1999. 5(11): p. 243-248.

32. Karpowicz, N., et al., Comparison between pulsed terahertz time-domain imaging and continuous wave terahertz imaging. Semiconductor Science and Technology, 2005. 20(7): p. S293-S299.

33. Lee, A.W. and Q. Hu, Real-time, continuous-wave terahertz imaging by use of a microbolometer focalplane array. Opt. Lett., 2005. 30(19): p. 2563-2565.

34. Ojefors, E., et al., A 0.65 THz Focal-Plane Array in a Quarter-Micron CMOS Process Technology. IEEE Journal of Solid-State Circuits, 2009. 44(7): p. 1968-1976.

35. Dobroiu, A., C. Otani, and K. Kawase, Terahertz-wave sources and imaging applications. Measurement Science \& Technology, 2006. 17(11): p. R161-R174.

36. Petkie, D.T., et al., Active and passive imaging in the THz spectral region: phenomenology, dynamic range, modes, and illumination. J. Opt. Soc. Am. B, 2008. 25(9): p. 1523-1531.

37. Adam, A.J.L., Review of Near-Field Terahertz Measurement Methods and Their Applications How to Achieve Sub-Wavelength Resolution at THz Frequencies. Journal of Infrared Millimeter and Terahertz Waves, 2011. 32(8-9): p. 976-1019.

38. Bitzer, A. and M. Walther, Terahertz near-field imaging of metallic subwavelength holes and hole arrays. Applied Physics Letters, 2008. 92(23): p. 231101.

39. Chen, H.T., R. Kersting, and G.C. Cho, Terahertz imaging with nanometer resolution. Applied Physics Letters, 2003. 83(15): p. 3009-3011.

40. Kersting, R., et al., Terahertz near-field microscopy, in Advances in Solid State Physics, R. Haug, Editor. 2008, Springer-Verlag Berlin: Berlin. p. 203-222.

41. Awad, M.M. and R.A. Cheville, Transmission terahertz waveguide-based imaging below the diffraction limit. Applied Physics Letters, 2005. 86(22): p. 221107.

42. Federici, J.F., et al., Terahertz imaging using an interferometric array. Applied Physics Letters, 2003. 83(12): p. 2477-2479.

43. Chan, W.L., J. Deibel, and D.M. Mittleman, Imaging with terahertz radiation. Reports on Progress in Physics, 2007. 70(8): p. 1325-1379.

44. Dorney, T., et al., Imaging with THz pulses, in 2000 International Conference on Image Processing, Vol I, Proceedings. 2000. p. 764-767.

45. Herrmann, M., R. Fukasawa, and O. Morikawa, Terahertz imaging. Terahertz Optoelectronics, 2005. 97: p. 331-381.

46. Jansen, C., et al., Terahertz imaging: applications and perspectives. Appl. Opt., 2010. 49(19): p. E48-E57.

47. Jepsen, P.U., D.G. Cooke, and M. Koch, Terahertz spectroscopy and imaging - Modern techniques and applications. Laser \& Photonics Reviews, 2011. 5(1): p. 124-166.

48. Mittleman, D.M., et al., Recent advances in terahertz imaging. Applied Physics B-Lasers and Optics, 1999. 68(6): p. 1085-1094.

49. Mickan, S., et al., Analysis of system trade-offs for terahertz imaging. Microelectronics Journal, 2000. 31(7): p. 503-514.

50. Planken, P.C.M., C. van Rijmenam, and R.N. Schouten, Opto-electronic pulsed THz systems. Semiconductor Science and Technology, 2005. 20(7): p. S121-S127.

51. Withayachumnankul, W., et al., T-Ray Sensing and Imaging. Proceedings of the IEEE, 2007. 95(8): p. 1528-1558.

52. Pickwell-Macpherson, E., et al., Recent developments of terahertz technology in biomedicine. Journal of Innovative Optical Health Sciences, 2008. 1(1): p. 29-44.

53. Baxter, J.B. and G.W. Guglietta, Terahertz Spectroscopy. Analytical Chemistry, 2011. 83(12): p. $4342-4368$.

54. Zhang, X.-C. and J. Xu, Introduction to THz wave photonics. 2009: Springer.

55. Mittleman, D.M., Imaging and sensing with terahertz radiation, in Review of Progress in Quantitative Nondestructive Evaluation, Vols $24 A$ and 24B, D.O. Thompson and D.E. Chimenti, Editors. 2005, Amer Inst Physics: Melville. p. 25-32.

56. Lee, Y.S., Principles of Terahertz Science and Technology, ed. Springer. 2009. 349.

57. Mittleman, D.M., Sensing with Terahertz Radiation, ed. Springer. 2003.

58. Pereira, M.F. and O. Shulika, Terahertz and Mid Infrared Radiation: Generation, Detection, and Applications (NATO Science for Peace and Security Series B: Physics and Biophysics). 2009.

59. Peiponen, K.-E.Z., Axel; Kuwata-Gonokami, Terahertz Spectroscopy and Imaging. Springer Series in Optical Sciences, Vol. 171, 2013. 
60. Dexheimer, S.L., Terahertz Spectroscopy: Principles and Applications, ed. T.F. Group. 2008.

61. Sakai, K., Terahertz optoelectronics. Terahertz Optoelectronics, Edited by K. Sakai. Berlin: Springer, 2005.1.

62. Katayama, I., et al., Ultrabroadband terahertz generation using 4- $N, N$-dimethylamino-4- $N$-methylstilbazolium tosylate single crystals. Applied Physics Letters, 2010. 97(2): p. 021105-3.

63. Cai, Y., et al., Coherent terahertz radiation detection: Direct comparison between free-space electro-optic sampling and antenna detection. Applied Physics Letters, 1998. 73(4): p. 444-446.

64. Kono, S., M. Tani, and K. Sakai, Ultrabroadband photoconductive detection: Comparison with free-space electro-optic sampling. Applied Physics Letters, 2001. 79(7): p. 898-900.

65. Karpowicz, N., et al., Coherent heterodyne time-domain spectrometry covering the entire "terahertz gap". Applied Physics Letters, 2008. 92(1): p. 011131.

66. Loffler, T., et al., Visualization and classification in biomedical terahertz pulsed imaging. Physics in Medicine and Biology, 2002. 47(21): p. 3847-3852.

67. Jepsen, P.U. and B. Fischer, Dynamic range in terahertz time-domain transmission and reflectionspectroscopy. Opt. Lett., 2005. 30(1): p. 29-31.

68. Jin, K.H., et al., High-speed terahertz reflection three-dimensional imaging for nondestructive evaluation. Opt. Express, 2012. 20(23): p. 25432-25440.

69. Bartels, A., et al., Ultrafast time-domain spectroscopy based on high-speed asynchronous optical sampling. Review of Scientific Instruments, 2007. 78(3): p. 035107-8.

70. Wilk, R., et al., OSCAT: Novel Technique for Time-Resolved Experiments Without Moveable Optical Delay Lines. Journal of Infrared, Millimeter, and Terahertz Waves, 2011. 32(5): p. 596-602.

71. Chan, W.L., et al., A single-pixel terahertz imaging system based on compressed sensing. Applied Physics Letters, 2008. 93(12): p. 121105.

72. Kohler, R., et al., Terahertz semiconductor-heterostructure laser. Nature, 2002. 417(6885): p. 156-159.

73. Lisauskas, A., et al., Terahertz imaging with GaAs field-effect transistors. Electronics Letters, 2008. 44(6): p. 408-409.

74. Knap, W., et al., Field Effect Transistors for Terahertz Detection: Physics and First Imaging Applications. Journal of Infrared Millimeter and Terahertz Waves, 2009. 30(12): p. 1319-1337.

75. El Fatimy, A., et al., Plasma wave field effect transistor as a resonant detector for 1 terahertz imaging applications. Optics Communications, 2009. 282(15): p. 3055-3058.

76. Dobroiu, A., et al., Terahertz imaging system based on a backward-wave oscillator. Applied Optics, 2004. 43(30): p. 5637-5646.

77. Knyazev, B.A., et al., Real-Time Imaging Using a High-Power Monochromatic Terahertz Source: Comparative Description of Imaging Techniques with Examples of Application. Journal of Infrared Millimeter and Terahertz Waves, 2011. 32(10): p. 1207-1222.

78. Li, Q., et al., Terahertz Computed Tomography Using A Continuous-Wave Gas Laser. Journal of Infrared Millimeter and Terahertz Waves, 2012. 33(5): p. 548-558.

79. Loeffler, T., et al., Continuous-wave terahertz imaging with a hybrid system. Applied Physics Letters, 2007. 90(9): p. 091111.

80. van der Valk, N.C.J., W.A.M. van der Marel, and P.C.M. Planken, Terahertz polarization imaging. Optics Letters, 2005. 30(20): p. 2802-2804.

81. Loffler, T., et al., Terahertz dark-field imaging of biomedical tissue. Optics Express, 2001. 9(12): p. 616621.

82. Lee, K. and J. Ahn, Single-pixel coherent diffraction imaging. Applied Physics Letters, 2010. 97(24): p. 241101.

83. Lee, K., et al., Coherent optical computing for T-ray imaging. Optics Letters, 2010. 35(4): p. 508-510.

84. Chan, W.L., et al., Terahertz imaging with compressed sensing and phase retrieval. Opt. Lett., 2008. 33(9): p. $974-976$.

85. Xu, Z. and E.Y. Lam, Image reconstruction using spectroscopic and hyperspectral information for compressive terahertz imaging. Journal of the Optical Society of America a-Optics Image Science and Vision, 2010. 27(7): p. 1638-1646.

86. Friederich, F., et al., THz Active Imaging Systems With Real-Time Capabilities. Terahertz Science and Technology, IEEE Transactions on, 2011. 1(1): p. 183-200.

87. Ruffin, A.B.., et al., Time reversal terahertz imaging. IEEE Journal of Quantum Electronics, 2002. 38(8): p. $1110-1119$.

88. Kiwa, T., et al., Laser terahertz-emission microscope for inspecting electrical faults in integrated circuits. Optics Letters, 2003. 28(21): p. 2058-2060.

89. Yamashita, M., et al., Imaging of large-scale integrated circuits using laser terahertz emission microscopy. Optics Express, 2005. 13(1): p. 115-120.

90. Johnson, J.L., T.D. Dorney, and D.M. Mittleman, Interferometric Imaging with terahertz pulses. IEEE Journal of Selected Topics in Quantum Electronics, 2001. 7(4): p. 592-599. 
91. Johnson, J.L., T.D. Dorney, and D.M. Mittleman, Enhanced depth resolution in terahertz imaging using phase-shift interferometry. Applied Physics Letters, 2001. 78(6): p. 835-837.

92. Kemp, M.C., et al. Security applications of terahertz technology. in Terahertz for Military and Security Applications. 2003. Orlando, FL, USA: SPIE.

93. Hoshina, H., et al., Noninvasive mail inspection system with terahertz radiation. Applied Spectroscopy, 2009. 63(1): p. 81-86.

94. Allis, D.G. and T.M. Korter, Theoretical analysis of the terahertz spectrum of the high explosive PETN. Chemphyschem, 2006. 7(11): p. 2398-2408.

95. Baker, C., et al., Detection of concealed explosives at a distance using terahertz technology. Proceedings of the IEEE, 2007. 95(8): p. 1559-1565.

96. Kemp, M.C., Explosives Detection by Terahertz Spectroscopy ;A Bridge Too Far? Terahertz Science and Technology, IEEE Transactions on, 2011. 1(1): p. 282-292.

97. Zhong, H., A. Redo-Sanchez, and X.C. Zhang, Identification and classification of chemicals using terahertz reflective spectroscopic focal-plane imaging system. Optics Express, 2006. 14(20): p. 9130-9141.

98. Federici, J.F., et al., THz imaging and sensing for security applications - explosives, weapons and drugs. Semiconductor Science and Technology, 2005. 20(7): p. S266-S280.

99. Kawase, K., Terahertz imaging for drug detection and large-scale integrated circuit inspection. Optics \& Photonics News, 2004. 15(10): p. 34-39.

100. Lu, M., et al., Detection and identification of illicit drugs using terahertz imaging. Journal of Applied Physics, 2006. 100(10).

101. Kawase, K., et al., Non-destructive terahertz imaging of illicit drugs using spectral fingerprints. Opt. Express, 2003. 11(20): p. 2549-2554.

102. Liu, H.B., et al., Terahertz spectroscopy and imaging for defense and security applications. Proceedings of the IEEE, 2007. 95(8): p. 1514-1527.

103. Appleby, R. and R.N. Anderton, Millimeter-wave and submillimeter-wave imaging for security and surveillance. Proceedings of the IEEE, 2007. 95(8): p. 1683-1690.

104. Jepsen, P.U., U. Moller, and H. Merbold, Investigation of aqueous alcohol and sugar solutions with reflection terahertz time-domain spectroscopy. Optics Express, 2007. 15(22): p. 14717-14737.

105. Rahani, E.K., et al., Mechanical Damage Detection in Polymer Tiles by THz Radiation. IEEE Sensors Journal, 2011. 11(8): p. 1720-1725.

106. Mousavi, P., et al., Simultaneous composition and thickness measurement of paper using terahertz timedomain spectroscopy. Appl. Opt., 2009. 48(33): p. 6541-6546.

107. Yasui, T., et al., Terahertz paintmeter for noncontact monitoring of thickness and drying progress in paint film. Applied Optics, 2005. 44(32): p. 6849-6856.

108. Wietzke, S., et al., Terahertz imaging: a new non-destructive technique for the quality control of plastic weld joints. Journal of the European Optical Society - Rapid publications; Vol 2 2007: p. 07013.

109. Nguema, E., et al., Dielectric properties of conducting polyaniline films by THz time-domain spectroscopy. European Polymer Journal, 2008. 44(1): p. 124-129.

110. Banerjee, D., et al., Diagnosing water content in paper by terahertz radiation. Opt. Express, 2008. 16(12): p. $9060-9066$.

111. Park, J.-W., et al., Terahertz spectroscopy approach of the fiber orientation influence on CFRP composite solid laminates. Journal of Mechanical Science and Technology, 2012. 26(7): p. 2051-2054.

112. Kawase, K., et al., THz imaging techniques for nondestructive inspections. Comptes Rendus Physique, 2010. 11(7-8): p. 510-518.

113. Seong-Tae, H., et al. Development of a compact sub-terahertz gyrotron and its application to t-ray real-time imaging for food inspection. in Infrared, Millimeter, and Terahertz Waves (IRMMW-THz), 2012 37th International Conference on. 2012.

114. Jördens, C. and M. Koch, Detection of foreign bodies in chocolate with pulsed terahertz spectroscopy. Optical Engineering, 2008. 47(3): p. 037003-037003.

115. Hor, Y.L., J.F. Federici, and R.L. Wample, Nondestructive evaluation of cork enclosures using terahertz/ millimeter wave spectroscopy and imaging. Applied Optics, 2008. 47(1): p. 72-78.

116. Zeitler, J.A., et al., Terahertz pulsed spectroscopy and imaging in the pharmaceutical setting - a review J. Pharm. Pharmacol., 2007. 59: p. 209.

117. Shen, Y.-C., Terahertz pulsed spectroscopy and imaging for pharmaceutical applications: A review. International Journal of Pharmaceutics, 2011. 417(1-2): p. 48-60.

118. King, M.D., P.M. Hakey, and T.M. Korter, Discrimination of chiral solids: A terahertz spectroscopic investigation of L-and DL-serine. The Journal of Physical Chemistry A, 2010. 114(8): p. 2945-2953.

119. Shen, Y.-C. and P.F. Taday, Development and application of terahertz pulsed imaging for nondestructive inspection of pharmaceutical tablet. IEEE Journal of Selected Topics in Quantum Electronics, 2008. 14(2): p. 407-415. 
120. Fitzgerald, A.J., B.E. Cole, and P.F. Taday, Nondestructive analysis of tablet coating thicknesses usign terahertz pulsed imaging. Journal of Pharmaceutical Sciences, 2005. 94(1): p. 177-183.

121. Maurer, L. and H. Leuenberger, Terahertz pulsed imaging and near infrared imaging to monitor the coating process of pharmaceutical tablets. International Journal of Pharmaceutics, 2009. 370: p. 8-16.

122. Ho, L., et al., Analysis of sustained-release tablet film coats using terahertz pulsed imaging. Journal of Controlled Release, 2007. 119(3): p. 253-261.

123. Mounaix, P., et al., Spectroscopy and terahertz imaging for sigillography applications. Journal of the European Optical Society-Rapid Publications vol 6 11002, 2011. 6: p. 1002.

124. Jackson, J.B., et al., Terahertz imaging for non-destructive evaluation of mural paintings. Optics Communications, 2008. 281(4): p. 527-532.

125. Abraham, E., et al., Broadband terahertz imaging of documents written with lead pencils. Optics Communications, 2009. 282(15): p. 3104-3107.

126. Adam, A.J.L., et al., TeraHertz imaging of hidden paintlayers on canvas. Opt. Express, 2009. 17(5): p. 3407-3416.

127. Fukunaga, K. and I. Hosako, Innovative non-invasive analysis techniques for cultural heritage using terahertz technology. Comptes Rendus Physique, 2010. 11(7-8): p. 519-526.

128. Fukunaga, K., et al., Application of terahertz spectroscopy for character recognition in a medieval manuscript. IEICE Electronics Express, 2008. 5(7): p. 223-228.

129. Oehrstroem, L., et al., Technical Note: Terahertz Imaging of Ancient Mummies and Bone. American Journal of Physical Anthropology, 2010. 142(3): p. 497-500.

130. Mittleman, D.M., et al., T-ray tomography. Optics Letters, 1997. 22(12): p. 904-906.

131. Zhang, X.C., Three-dimensional terahertz wave imaging. Philosophical Transactions of the Royal Society of London Series a-Mathematical Physical and Engineering Sciences, 2004. 362(1815): p. 283-298.

132. Wang, S. and X.C. Zhang, Pulsed terahertz tomography. Journal of Physics D: Applied Physics, 2004. 37(4): p. R1.

133. Semenov, S.Y., et al., Three-dimensional microwave tomography: initial experimental imaging of animals. Biomedical Engineering, IEEE Transactions on, 2002. 49(1): p. 55-63.

134. Nguyen, K.L., et al., Three-dimensional imaging with a terahertz quantum cascade laser. Optics Express, 2006. 14(6): p. 2123-2129.

135. Kato, E., et al., 3D Spectroscopic Computed Tomography Imaging Using Terahertz Waves. 35th International Conference on Infrared, Millimeter, and Terahertz Waves. 2010.

136. Wallace, V.P., et al., Three-dimensional imaging of optically opaque materials using nonionizing terahertz radiation. Journal of the Optical Society of America a-Optics Image Science and Vision, 2008. 25(12): p. 3120-3133.

137. Gbur, G. and E. Wolf, Relation between computed tomography and diffraction tomography. JOSA A, 2001. 18(9): p. 2132-2137.

138. Ferguson, B., et al., T-ray computed tomography. Optics Letters, 2002. 27(15): p. 1312-1314.

139. Chen, K. and D.A. Castanon, Robust Multifrequency Inversion in Terahertz Diffraction Tomography, in Computational Imaging Ix, C.A. Bouman, I. Pollak, and P.J. Wolfe, Editors. 2011.

140. Jin, K.H., et al., Compressed sensing pulse-echo mode terahertz reflectance tomography. Optics Letters, 2009. 34(24): p. 3863-3865.

141. Sunaguchi, N., et al., Depth-resolving THz imaging with tomosynthesis. Optics Express, 2009. 17(12): p. 9558-9570.

142. Niklason, L.T., et al., Digital tomosynthesis in breast imaging. Radiology, 1997. 205(2): p. 399-406.

143. Takayanagi, J., et al., High-resolution time-of-flight terahertz tomography using a femtosecond fiber laser. Optics Express, 2009. 17(9): p. 7549-7555.

144. Hirsch, O., P. Alexander, and L.F. Gladden, Techniques for cancellation of interfering multiple reflections in terahertz time-domain measurements. Microelectronics Journal, 2008. 39(5): p. 841-848.

145. Kitahara, H., et al., THz tomographic imaging by using two-dimensional electro-optic sampling. 2007 Joint 32nd International Conference on Infrared and Millimeter Waves and 15th International Conference on Terahertz Electronics, Vols 1 and 2. 2007. 892-893.

146. Kitahara, H., M. Tani, and M. Hangyo, Three-Dimensional Tomographic Imaging in Terahertz Region. Japanese journal of applied physics, 2010. 49(2).

147. Cho, S.-H., et al., Fast terahertz reflection tomography using block-based compressed sensing. Optics Express. 19(17): p. 16401-16409.

148. Takayanagi, J., et al., High-resolution time-of-flight terahertz tomography using a femtosecond fiber laser. Optics express, 2009. 17(9): p. 7533-7539.

149. Brucherseifer, M., et al., Angle-dependent THz tomography-characterization of thin ceramic oxide films for fuel cell applications. Applied Physics B: Lasers and Optics, 2001. 72(3): p. 361-366. 
150. Schwerdtfeger, M., et al., Beating the wavelength limit: three-dimensional imaging of buried subwavelength fractures in sculpture and construction materials by terahertz time-domain reflection spectroscopy. Applied Optics, 2013. 52(3): p. 375-380.

151. Pearce, J., et al., T-ray reflection computed tomography. 2005 Conference on Lasers \& Electro-Optics. 2005. 2120-2122.

152. Pearce, J., et al., Terahertz wide aperture reflection tomography. Optics Letters, 2005. 30(13): p. 1653-1655.

153. Matoba, O., et al., Real-time three-dimensional object reconstruction by use of a phase-encoded digital hologram. Applied Optics, 2002. 41(29): p. 6187-6192.

154. Zhang, Y., et al., Terahertz digital holography. Strain, 2008. 44(5): p. 380-385.

155. Ruffin, A.B.., et al., Time reversal and object reconstruction with single-cycle pulses. Optics letters, 2001. 26(10): p. 681-683.

156. Mahon, R.J., J.A. Murphy, and W. Lanigan, Digital holography at millimetre wavelengths. Optics Communications, 2006. 260(2): p. 469-473.

157. McAuley, I., et al., Applications of holography in the millimeter wave and terahertz region, in Terahertz Technology and Applications IV, L.P. Sadwick and C.M.M. Osullivan, Editors. 2011.

158. Ding, S.-H., et al., Continuous-wave terahertz digital holography by use of a pyroelectric array camera. Optics letters, 2011. 36(11): p. 1993-1995.

159. Knyazev, B.A., et al., Classic holography, tomography and speckle metrology using a high-power terahertz free electron laser and real-time image detectors. 35th International Conference on Infrared, Millimeter, and Terahertz Waves. 2010.

160. Li, Q., et al., Research on Reconstruction Algorithms in $2.52 \mathrm{THz}$ Off-axis Digital Holography. Journal of Infrared Millimeter and Terahertz Waves, 2012. 33(10): p. 1039-1051.

161. Li, Q., et al., Experimental research on resolution improvement in CW THz digital holography. Applied Physics B-Lasers and Optics, 2012. 107(1): p. 103-110.

162. Jahns, J. and S.J. Walker, Two-dimensional array of diffractive microlenses fabricated by thin film deposition. Applied optics, 1990. 29(7): p. 931-936.

163. Wang, S. and X.-C. Zhang, Tomographic imaging with a terahertz binary lens. Applied physics letters, 2003. 82(12): p. 1821-1823.

164. Walsby, E., et al., Multilevel silicon diffractive optics for terahertz waves. Journal of Vacuum Science \& Technology B: Microelectronics and Nanometer Structures, 2002. 20(6): p. 2780-2783.

165. Wang, S., et al., Characterization of T-ray binary lenses. Optics letters, 2002. 27(13): p. 1183-1185.

166. McClatchey, K., M.T. Reiten, and R.A. Cheville, Time resolved synthetic aperture terahertz impulse imaging. Applied Physics Letters, 2001. 79(27): p. 4485-4487.

167. Henry, S.C., et al., Three-dimensional broadband terahertz synthetic aperture imaging. Optical Engineering, 2012. 51(9).

168. O'Hara, J. and D. Grischkowsky, Quasi-optic synthetic phased-array terahertz imaging. Journal of the Optical Society of America B-Optical Physics, 2004. 21(6): p. 1178-1191.

169. Heimbeck, M.S., et al., Terahertz interferometric synthetic aperture tomography for confocal imaging systems. Optics Letters, 2012. 37(8): p. 1316-1318.

170. Bandyopadhyay, A., et al., Terahertz interferometric and synthetic aperture imaging. Journal of the Optical Society of America A, 2006. 23(5): p. 1168-1178.

171. O'Hara, J. and D. Grischkowsky, Synthetic phased-array terahertz imaging. Optics letters, 2002. 27(12): p. 1070-1072.

172. Buma, T. and T.B. Norris, Time reversal three-dimensional imaging using single-cycle terahertz pulses. Applied Physics Letters, 2004. 84(12): p. 2196-2198.

173. Dorney, T.D., et al., Terahertz reflection imaging using Kirchhoff migration. Optics Letters, 2001. 26(19): p. 1513-1515.

174. Musheinesh, M.A., et al., Time-reversal and model-based imaging in a THz waveguide. Opt. Express, 2009. 17(16): p. 13663-13670.

175. Brahm, A., et al., Vlumetric spectral analysis of materials using terahertz-tomography techniques. Applied Physics B-Lasers and Optics, 2010. 100(1): p. 151-158.

176. Jewariya, M., et al., Fast three-dimensional terahertz computed tomography using real-time line projection of intense terahertz pulse. Opt. Express, 2013. 21(2): p. 2423-2433.

177. Ewers, B., et al., Terahertz Spectral Computed Tomography. 2009 34th International Conference on Infrared, Millimeter, and Terahertz Waves, Vols 1 and 2. 2009. 138-139.

178. Toft, P., The Radon Transform : Theory and Implementation, " Ph.D. dissertation, Department of Mathematical Modelling, Section for Digital Signal Processing, Technical University of Denmark, 1996. . 1996.

179. Radon, J., Uber die Bestimmung von Funktionen durch ihre In- tegralwerte langs gewisser Mannigfaltigkeiten.” Ber. Ver. Sachs. Akad. Wiss. Leipzig, in German. An english translation can be found 
in S. R. Deans : The Radon Transform and Some of Its Applications. . Math-Phys. K1, vol. 69, pp. 262-277, April 1917.

180. Hounsfield, G.N., Computerized transverse axial scanning (tomography): Part 1. Description of system. British Journal of Radiology, 1973. 46(552): p. 1016-1022.

181. Ter-Pogossian, M.M., et al., A positron-emission transaxial tomograph for nuclear imaging (PETT). Radiology, 1975. 114(1): p. 89-98.

182. Toft, P.A., Iterative Methods for Reconstructing PET Images. 1996.

183. Recur, B., et al., Investigation on reconstruction methods applied to $3 D$ terahertz computed tomography. Optics Express, 2011. 19(6): p. 5105-5117.

184. Shepp, L.A. and B.F. Logan, The Fourier reconstruction of a head section. IEEE Trans. Nucl. Sci, 1974. 21(3): p. 21-43.

185. Andersen, A.H., Application of ray tracing towards a correction for refracting effects in computed tomography with diffracting sources. :Thesis (Ph. D.), 1983(Purdue Univ.,Lafayette, IN).

186. Shepp, L.A. and Y. Vardi, Maximum likelihood reconstruction for emission tomography. Medical Imaging, IEEE Transactions on, 1982. 1(2): p. 113-122.

187. Kuba, A. and G. Hermann, Discrete Tomography: Foundations, Algorithms and Applications, 1999, Birkhauser.

188. Katz, M.B., Questions of uniqueness and resolution in reconstruction from projections. 1978: SpringerVerlag.

189. Averbuch, A., et al., Fast and accurate polar Fourier transform. Applied and Computational Harmonic Analysis, 2006. 21(2): p. 145-167.

190. Jones, R., T. Cooke, and N.J. Redding, Implementation of the Radon Transform Using Non-equispaced Discrete Fourier Transforms, 2004, DTIC Document.

191. Potts, D. and G. Steidl. New Fourier reconstruction algorithms for computerized tomography. in International Symposium on Optical Science and Technology. 2000. International Society for Optics and Photonics.

192. Gordon, R., A Tutorial on ART (Algebraic Reconstruction Techniques. IEEE Trans. Nucl. Sci. NS-21, 1974(3): p. 78-93.

193. Gordon, R., R. Bender, and G.T. Herman, Algebraic reconstruction techniques (ART) for three-dimensional electron microscopy and x-ray photography. Journal of theoretical Biology, 1970. 29(3): p. 471.

194. Herman, G.T., Image reconstruction from projections. Real-Time Imaging, 1995. 1(1): p. 3-18.

195. Herman, G.T. and L.B. Meyer, Algebraic reconstruction techniques can be made computationally efficient [positron emission tomography application]. Medical Imaging, IEEE Transactions on, 1993. 12(3): p. 600-609.

196. Lent, A. Maximum entropy and multiplicative ART. in Proc. Conf. Image Analysis and Evaluation, SPSE, Toronto. 1976.

197. Lu, W. and F.-F. Yin, Adaptive algebraic reconstruction technique. Medical physics, 2004. 31: p. 3222.

198. Gilbert, P., Iterative methods for the three-dimensional reconstruction of an object from projections. Journal of Theoretical Biology, 1972. 36(1): p. 105.

199. Mumcuoglu, E., et al., Fast gradient-based methods for Bayesian reconstruction of transmission and emission PET images. Medical Imaging, IEEE Transactions on, 1994. 13(4): p. 687-701.

200. Kaufman, L., Maximum likelihood, least squares, and penalized least squares for PET. Medical Imaging, IEEE Transactions on, 1993. 12(2): p. 200-214.

201. Recur, B., et al., Terahertz radiation for tomographic inspection. Optical Engineering, 2012. 51(9): p. 091609-1-091609-7.

202. Oden, J., et al., Imaging of broadband terahertz beams using an array of antenna-coupled microbolometers operating at room temperature. Opt. Express, 2013. 21(4): p. 4817-4825. 